\title{
Extensive Inhibitory Gating of Viscerosensory Signals by a Sparse Network of Somatostatin Neurons
}

\author{
(Dimberly R. Thek, ${ }^{1}$ Sarah J.M. Ong, ${ }^{2}$ David C. Carter, ${ }^{2}$ Jaspreet K. Bassi, ${ }^{2}{ }^{-}$Andrew M. Allen, ${ }^{1,2}$ \\ and ${ }^{\circledR S t u a r t} \mathrm{~J}$. McDougall $^{1}$ \\ ${ }^{1}$ Florey Institute of Neuroscience and Mental Health, and ${ }^{2}$ Department of Physiology, University of Melbourne, Parkville, Victoria 3010, Australia
}

Integration and modulation of primary afferent sensory information begins at the first terminating sites within the CNS, where central inhibitory circuits play an integral role. Viscerosensory information is conveyed to the nucleus of the solitary tract (NTS) where it initiates neuroendocrine, behavioral, and autonomic reflex responses that ensure optimal internal organ function. This excitatory input is modulated by diverse, local inhibitory interneurons, whose functions are not clearly understood. Here we show that, in male rats, $65 \%$ of somatostatin-expressing (SST) NTS neurons also express GAD67, supporting their likely role as inhibitory interneurons. Using wholecell recordings of NTS neurons, from horizontal brainstem slices of male and female SST-yellow fluorescent protein (YFP) and SSTchannelrhodopsin 2 (ChR2)-YFP mice, we quantified the impact of SST-NTS neurons on viscerosensory processing. Light-evoked excitatory photocurrents were reliably obtained from SST-ChR2-YFP neurons $(n=16)$ and the stimulation-response characteristics determined. Most SST neurons (57\%) received direct input from solitary tract (ST) afferents, indicating that they form part of a feedforward circuit. All recorded SST-negative NTS neurons $(n=72)$ received SST-ChR2 input. ChR2-evoked PSCs were largely inhibitory and, in contrast to previous reports, were mediated by both GABA and glycine. When timed to coincide, the ChR2-activated SST input suppressed ST-evoked action potentials at second-order NTS neurons, demonstrating strong modulation of primary viscerosensory input. These data indicate that the SST inhibitory network innervates broadly within the NTS, with the potential to gate viscerosensory input to powerfully alter autonomic reflex function and other behaviors.

Key words: GABA; GAD67; glycine; somatostatin; vagus; viscerosensory

Significance Statement

Sensory afferent input is modulated according to state. For example the baroreflex is altered during a stress response or exercise, but the basic mechanisms underpinning this sensory modulation are not fully understood in any sensory system. Here we demonstrate that the neuronal processing of viscerosensory information begins with synaptic gating at the first central synapse with second-order neurons in the NTS. These data reveal that the somatostatin subclass of inhibitory interneurons are driven by visceral sensory input to play a major role in gating viscerosensory signals, placing them within a feedforward circuit within the NTS.

\section{Introduction}

The majority of visceral sensory afferent neurons, whose axons contribute to the vagus and glossopharyngeal nerves, synapse in

\footnotetext{
Received Nov. 28, 2018; revised Aug. 7, 2019; accepted Aug. 10, 2019

Author contributions: A.M.A. and S.J.M. designed research; K.R.T., S.J.M.O., D.C.C., J.K.B., and S.J.M. performed research; K.R.T. and S.J.M. analyzed data; K.R.T., A.M.A., and S.J.M. wrote the paper.

This work was supported by Grants from the Australian Research Council (Discovery Project 170104861 and 170104582; S.J.M., A.M.A.), the National Health and Medical Research Council of Australia (APP1120477; A.M.A.), University of Melbourne Early Career Researcher Grants Scheme (S.J.M.) and the Victorian Government's Operational Infrastructure Support Program (Florey).

The authors declare no competing financial interests.

Correspondence should be addressed to Stuart J. McDougall at stuart.mcdougall@florey.edu.au or Andrew M. Allen at a.allen@unimelb.edu.au.

https://doi.org/10.1523/JNEUROSCI.3036-18.2019

Copyright $\odot 2019$ the authors
}

the nucleus of the solitary tract (NTS; Janig, 2006). This viscerosensory input drives autonomic reflexes, and also modulates neuroendocrine function and behaviors, including satiety and reward (Andresen and Paton, 2011; Han et al., 2018). Intermixed with the excitatory vagal afferent terminals within the NTS is an intrinsic inhibitory network (Fong et al., 2005; Okada et al., 2008; McDougall and Andresen, 2012). Spontaneous miniature IPSCs are recorded in all NTS neurons (Jin et al., 2004; McDougall and Andresen, 2012), indicating that this inhibitory network likely modulates the integration of primary sensory input. Within the cardiorespiratory NTS, GABAergic neurons are the proposed primary target of somatic afferent terminals that are activated during locomotion (Potts, 2002), and are implicated in the coordination of baroreceptor reflex resetting during exercise (Potts, 
2006; Michelini, 2007). Despite the current literature indicating an important role for inhibition within the NTS, the specific mechanisms of inhibition at the synaptic to local network level remains ill defined. In part this lack of knowledge is because of the limitations in selectively activating inhibitory NTS neurons in the absence of pharmacological agents (McDougall and Andresen, 2012).

Neurons expressing somatostatin (SST) are a major subclass of inhibitory neurons, best characterized in the context of cortical function where they constitute one-third of inhibitory interneurons and exhibit a high level of spontaneous spiking activity (3-10 Hz; Urban-Ciecko and Barth, 2016). Throughout different nuclei within the CNS, SST inhibitory neurons are diverse in their coexpression of other molecular markers, such as calbindin (Yavorska and Wehr, 2016). Cortical and hippocampal SST neurons are exclusively GABAergic (Urban-Ciecko and Barth, 2016). Yet in the dorsal horn of the spinal cord they are mostly (94\%) excitatory (Duan et al., 2014) and in the pre-Bötzinger complex of the brainstem, a proportion of SST neurons are also excitatory (Tan et al., 2008; Tupal et al., 2014; Cui et al., 2016). Throughout the NTS published evidence indicates that most SST neurons are inhibitory (Wang and Bradley, 2010; Lewin et al., 2016). Signal transduction from primary afferents to second-order NTS neurons is very efficient in part because of the high probability of release at these terminals (Bailey et al., 2006; Peters et al., 2008). Thus, gain control via inhibitory synapses at second-order neurons likely serves an important modulatory role in transfer of sensory signals to higher brain areas. Here SST inhibitory interneurons may form part of a microcircuit "gate" that controls the flow of sensory information through, and from, the NTS.

In this study we mapped SST neurons within the NTS and defined near two-thirds as expressing glutamic acid decarboxylase 67 (GAD1; termed GAD67 in this study), with the remainder expressing the vesicular glutamate transporter 2 (vGlut; Slc17a6) mRNA. We studied this network of neurons within the NTS using transgenic mice in which enhanced yellow fluorescent protein (YFP) or channelrhodopsin 2 (ChR2) fused to YFP, was expressed in all SST neurons. We find that the majority (57\%) of NTS SST neurons are directly driven by vagal afferent input. The SST NTS neurons use both GABA and glycine to alter viscerosensory signal throughput at SST-negative NTS neurons. Despite constituting a relatively small population of neurons within the NTS, all SST-negative neurons studied received input from SST neurons and, in some cases, the SST input disinhibited other SST neurons. The SST neurons studied are not a source of GABA input to presynaptic vagal afferent terminals, which are known to express $\mathrm{GABA}_{B}$ receptors. Finally, our data indicate that the SST neuronal network modulates viscerosensory information by synaptic gating at the earliest stages of sensory reflex processing within the CNS.

\section{Materials and Methods}

All animal procedures were approved by the Animal Ethics Committees at the Florey Institute of Neuroscience and Mental Health and University of Melbourne and were performed in accordance with the Prevention of Cruelty to Animals Act 1986 under the guidelines of the National Health and Medical Research Council "Australian code for the care and use of animals for scientific purposes” (Ed 8).

Animal models. Six male Sprague-Dawley rats $(\sim 320 \mathrm{~g})$ were used to examine the distribution of NTS neurons expressing mRNA for SST, GAD67, vGlut, and vesicular GABA transporter (VGAT; Slc32a1) using RNAscope [Advanced Cell Diagnostics (ACD)]. We used two transgenic mouse lines derived from cross-breeding an SST-Cre recombinase (Cre) line; B6N;Cg-Sst ${ }^{\text {tm2.1(cre)Zjh } / J ~[T h e ~ J a c k s o n ~ L a b o r a t o r y ~(J A X)] . ~ O n e ~ l i n e ~}$ was created by a cross with B6.129X1-Gt(ROSA)26Sor ${ }^{\text {tm1 } 1(E Y F P) C o s} / \mathrm{J}$ mice (JAX) and are described in the text as SST-YFP mice $(n=5$ female, 1 male). The second line were derived from a cross with B6;129SGt(ROSA)26Sor ${ }^{\left.\text {tm32(CAG-COP4 }{ }^{*} H 134 R / E Y F P\right) H z e} / \mathrm{J}$ mice (JAX) and their offspring are referred to as SST-ChR2-YFP mice in the text ( $n=46$ female, 37male).

RNA scope for SST, GAD67, and vGlut mRNA. Rats were deeply anesthetized by inhalation of isoflurane. Brains were quickly extracted, frozen in isopentane equilibrated with dry ice, and then stored at $-80^{\circ} \mathrm{C}$ until further processing. Sixteen $\mu \mathrm{m}$ coronal sections of the brainstem were cut in a cryostat $\left(-20^{\circ} \mathrm{C}\right.$; Leica Microsystems) and thaw-mounted onto Superfrost-Plus slides (ThermoFisher Scientific). Sections were left to dry within the cryostat for at least $30 \mathrm{~min}$ before storing at $-80^{\circ} \mathrm{C}$. For RNAscope processing, frozen sections were immediately fixed in $4 \%$ paraformaldehyde (PFA) for $16 \mathrm{~min}$ at $4^{\circ} \mathrm{C}$, rinsed twice, for $1 \mathrm{~min}$ each, in DEPC-treated PBS and then dehydrated in 50, 70, and 100\% ethanol solutions ( 5 min each concentration). The dehydrated sections were then stored in $100 \%$ ethanol overnight at $-20^{\circ} \mathrm{C}$. On the following day, slides were air dried at room temperature (RT) and hydrophobic barriers were created around each section using ImmEdge hydrophobic PAP pen (Vector Laboratories). Once the barrier had dried the sections were incubated with Protease IV (ACD, catalog \#322336) for $20 \mathrm{~min}$ at RT, rinsed twice with DEPC-treated PBS for 1 min each before incubating with the probe mixture for $2 \mathrm{~h}$ at $40^{\circ} \mathrm{C}$. The probe mixture consisted of probes against SST (ACD, catalog \#412181-C3), GAD67 (ACD, catalog \#316401-C2), VGAT (ACD, catalog \#424541-C2) and vGlut (ACD, catalog \#317011-C2) diluted in probe diluent (ACD, catalog \#300041). After probe incubation, slides were rinsed twice with $1 \times$ wash buffer $(A C D$, catalog \#310091) for $2 \mathrm{~min}$ each, before signal amplification was achieved through multiple amplification steps using the RNAscope multiplex fluorescence kit (ACD, catalog \#320851) according to the manufacturer's protocol. Sections were incubated in a series of amplifier reagents at $40^{\circ} \mathrm{C}$ : AMP1 (ACD, catalog \#320852) for $30 \mathrm{~min}$, AMP2 (ACD, catalog \#320853) for $15 \mathrm{~min}$, AMP3 (ACD, catalog \#320854) for $30 \mathrm{~min}$, while washing slides twice in between each amplifying step with $1 \times$ wash buffer for 2 min each. Finally, sections were incubated with AMP4 AltA (ACD, catalog \#320855) for $15 \mathrm{~min}$ at $40^{\circ} \mathrm{C}$, before rinsing twice with $1 \times$ wash buffer for 2 min each and staining with DAPI (ACD, catalog \#320858) for 20 s. They were coverslipped using ProLong Gold Antifade Mountant (ThermoFisher Scientific) and left to dry at RT before storing at $4^{\circ} \mathrm{C}$ and imaging.

Immunohistochemical localization of ChR2-YFP. Immunohistochemistry to visualize ChR2-YFP expression in the brainstem, was performed in adult mice $(n=2$ female, $461 \mathrm{~d}$ old; $n=1$ male, $349 \mathrm{~d}$ old, $32.9 \pm$ $3.38 \mathrm{~g})$. The mice were anesthetized with $5 \%$ isoflurane and perfused transcardially with $40 \mathrm{ml} 0.1 \mathrm{M}$ phosphate buffer solution (PBS; $0.1 \mathrm{M}, \mathrm{pH}$ 7.4; in mM: 7.70 $\mathrm{Na}_{2} \mathrm{HPO}_{4}, 2.7 \mathrm{NaH}_{2} \mathrm{PO}_{4}, 15.40 \mathrm{NaCl}$ ) and $40 \mathrm{ml}$ of $4 \%$ (w/v in $0.1 \mathrm{M}$ PBS) PFA. Brainstems were postfixed in $4 \%$ PFA for $4 \mathrm{~h}$, then transferred to sucrose solution (30\% sucrose in $0.1 \mathrm{M}$ PBS) and left overnight at $4^{\circ} \mathrm{C}$. Thirty-micrometer-thick coronal sections were cut on a freezing microtome (Leica Microsystems). After two rinses in PBS (10 min each), sections were incubated overnight at room temperature in 0.1 M PBS containing chicken anti-GFP (1:10,000; ab13970, Abcam) and Triton X-100 (0.3\%), then incubated at RT for $2 \mathrm{~h}$ in goat anti-chicken AlexaFluor 488 (1:500; catalog\#A32931, ThermoFisher Scientific) and Triton X-100 (0.3\%) in $0.1 \mathrm{M}$ PBS. Sections were mounted on gelatincoated slides and coverslipped using Slow Fade Gold (ThermoFisher Scientific). Sections were scanned using a confocal microscope (LSM 780, Carl Zeiss) and images processed using ImageJ software (NIH).

Horizontal brainstem slice preparation. Brainstem slices were prepared from both male and female SST-YFP $[n=6 ; 41 \pm 1 \mathrm{~d}$ old $(38-45 \mathrm{~d}$ range), $22.0 \pm 0.8 \mathrm{~g}, 5$ female $]$ and SST-ChR2-YFP mice $[n=80 ; 260 \pm$ $162 \mathrm{~d}$ old (32-585 d range), $31.4 \pm 9.7 \mathrm{~g}, 44$ female]. No differences were found related to sex or age. Mice were deeply anesthetized with $5 \%$ Isoflurane and the medulla removed, blocked and rapidly cooled in artificial CSF $\left[\mathrm{aCSF} ; 2^{\circ} \mathrm{C}\right.$, containing the following (in mM): $125 \mathrm{NaCl}, 3 \mathrm{KCl}$, $1.2 \mathrm{KH}_{2} \mathrm{PO}_{4}, 1.2 \mathrm{MgSO}_{4}, 25 \mathrm{NaHCO}_{3}, 10$ dextrose, $2 \mathrm{CaCl}_{2}(300$ $\mathrm{mOsm})$. A wedge of tissue from the rostral ventral surface of the medulla 
was removed enabling $250 \mu \mathrm{m}$ horizontal slices of the dorsal medulla that contained both the solitary tract (ST) and NTS to be cut on a vibratome (VT1200s, Leica Microsystems). The slice was continuously perfused in aCSF solution, bubbled with $95 \% \mathrm{O}_{2}, 5 \% \mathrm{CO}_{2}$, at $32^{\circ} \mathrm{C}$.

Whole-cell recordings and neuron selection. Recording pipettes $(2.4-6.3 \mathrm{M} \Omega$ ) contained either a low $\mathrm{Cl}^{-}$internal solution $\left(10 \mathrm{mM} \mathrm{Cl}^{-}\right)$ containing the following (in $\mathrm{mm}$ ): $6 \mathrm{NaCl} ; 4$ $\mathrm{NaOH} ; 130 \mathrm{KOH}$, potassium gluconate; 11 EGTA; $1 \mathrm{CaCl}_{2} ; 10$ HEPES; $1 \mathrm{MgCl}_{2} ; 0.1 \%$ biocytin, pH $7.3(290 \mathrm{mOsm})$ or a high $\mathrm{Cl}^{-}$internal solution $\left(54 \mathrm{mM} \mathrm{Cl}^{-}\right.$) containing the following (in $\mathrm{mm}$ ): $10 \mathrm{NaCl} ; 40 \mathrm{KCl} ; 90 \mathrm{KOH}$, potassium gluconate; $11 \mathrm{EGTA} ; 1 \mathrm{CaCl}_{2} ; 10$ HEPES; $1 \mathrm{MgCl}_{2} ; 0.1 \%$ biocytin, $\mathrm{pH} 7.3$ (290 mOsm). As a consequence $\mathrm{E}_{\mathrm{Cl}}$ was -68.8 and $-24.7 \mathrm{mV}$ for 10 and $54 \mathrm{~mm} \mathrm{Cl}^{-}$internal solutions, respectively. Pipettes were visually guided to NTS neurons using a fixed stage scope (Zeiss Examiner) and camera (Rolera EM-C2, Q-Imaging). Whole-cell recordings were made in either voltage-clamp [holding voltage $\left(V_{\mathrm{H}}\right)-60$ or $-40 \mathrm{mV}$ ] or currentclamp mode (MultiClamp 700B and pClamp 10.3, Molecular Devices). We were able to distinguish IPSCs from EPSCs using the low $\mathrm{Cl}^{-}$ internal solution $\left(10 \mathrm{mM} \mathrm{Cl}^{-}\right)$with a $\mathrm{V}_{\mathrm{H}}-40$ $\mathrm{mV}$; where chloride currents were outward and sodium current inward. Signals were sampled at $20 \mathrm{kHz}$ and filtered at $10 \mathrm{kHz}$. Liquid junction potentials were not corrected $(-6.2 \mathrm{mV}$ at $32^{\circ} \mathrm{C}$ ). In 16 neurons YFP was visualized to target SST neurons for recording (see Fig. 2) but otherwise all other neurons (see Figs. 3-8) were selected without imaging YFP within the medial/caudal NTS.

LED activation of ChR2 and electrical activation of primary afferents in the solitary tract. Pulses of light (465 nm, $10 \mathrm{~mW}$ at fiber tip) from a light emitting diode (LED, Plexon) were delivered via fiber optic affixed to a micromanipulator and positioned to illuminate the ipsilateral NTS. A range of light pulses (1-10) at varying durations $(0.2-100 \mathrm{~ms})$ and frequencies $(5-50 \mathrm{~Hz})$ were used. Inward currents that exhibited no latency to onset, i.e., synchronous with the light pulse, and began to decay synchronous with the LED pulse terminating, defined ChR2 photocurrents. This characteristic response identified SST NTS neurons from those not expressing ChR2. Bath-applied antagonists for $\mathrm{GABA}_{\mathrm{A}}$ receptors, gabazine (SR95531, $3 \mu \mathrm{M}$; Tocris Bioscience, In Vitro Technologies); glycine receptors, strychnine (1 $\mu \mathrm{M}$, Sigma-Aldrich); and AMPA receptors, NBQX (20 $\mu \mathrm{M}$; Tocris Bioscience) were used to define neurotransmitter/receptor mechanisms.

Primary afferent axons within the ST were activated by electric stimulation using a concentric bipolar electrode (125 $\mu \mathrm{m}$ outer diameter; FHC) that was placed on the visible ST at a distance of 1-2 $\mathrm{mm}$ from recorded neurons. Trains of five shocks $(100 \mu \mathrm{s})$ at $20 \mathrm{~Hz}$, where intensity was varied (0.03-0.5 mA), evoked ST-EPSCs and ST-initiated action potentials (APs) in NTS neurons. NTS neurons were classed as a secondorder neuron, directly receiving primary afferent input, if ST-evoked EPSCs exhibited a synaptic jitter (SD of EPSC latency) $<200 \mu$ s and frequency-dependent depression (Peters et al., 2011). Otherwise neurons were classified as higher order. If ST stimulation did not elicit a response, neurons were considered "not connected".
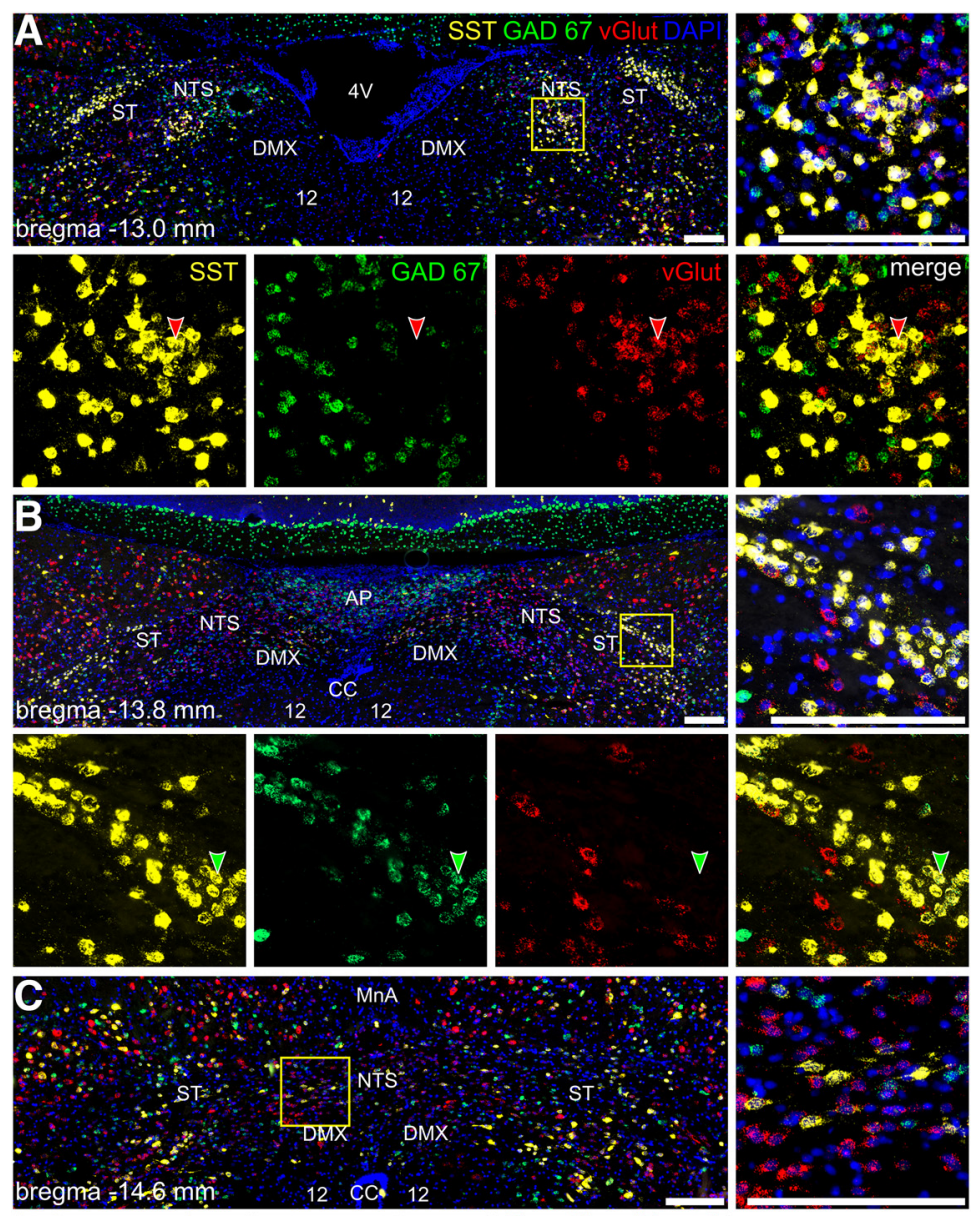

Figure 1. SST mRNA-expressing neurons also express mRNA for GAD67 or vGlut within the NTS. Fluorescence micrographs showing expression of mRNA for SST (yellow), GAD67 (green), vGlut (red), and DAPI (blue) at three different rostrocaudal levels of , Rostral to area postrema; $\boldsymbol{B}$, mid area postrema; $\boldsymbol{C}$, the commissural NTS. The yellow, boxed areas indicate the area for medulla. D, Neurons were counted within the NTS at these three levels (including the parasolitary nucleus). The stacked histograms show the number of neurons of each neurochemical phenotype (mean \pm SEM, derived from 6 animals).

Statistical analysis. Within the NTS we counted mRNA expressing cells across three levels of the NTS in six animals. For whole-cell recordings input resistance was calculated from the steady-state current change to a $+10 \mathrm{mV}$ step. Resting membrane potential from a $>5 \mathrm{~s}$ period of gap free recording at $I=0 \mathrm{pA}$, during which there were no action potentials. Spontaneous AP frequency was measured over $60 \mathrm{~s}$ at $I=0 \mathrm{pA}$ in active neurons. Rheobase was measured at the current injection that elicited an AP in $I-F$ protocols. Frequency accommodation was calculated as the ratio of Fmax, the reciprocal of the first interspike interval of a $+80 \mathrm{pA}$ step, to Fsteady, the reciprocal of the interpike interval of the final four APs to the same $400 \mathrm{~ms}+80 \mathrm{pA}$ step (Ma et al., 2006). The AP half-width was measured from the average of five spontaneous APs (Stimfit). The Sag-V slope was calculated as the difference between steady-state $V_{\mathrm{m}}$ and the peak $V_{\mathrm{m}}$ within $100 \mathrm{~ms}$ of hyperpolarizing steps ( -60 to $0 \mathrm{pA}$ from holding, in $10 \mathrm{pA}$ increments). Where the Sag-V slope is the slope of the regression from the resulting $I-V$ curve. ChR2-evoked PSC characteristics; including amplitude, latency, and decay were measured from at least 

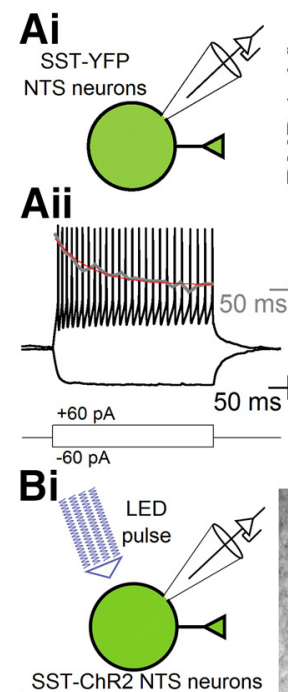

Bii
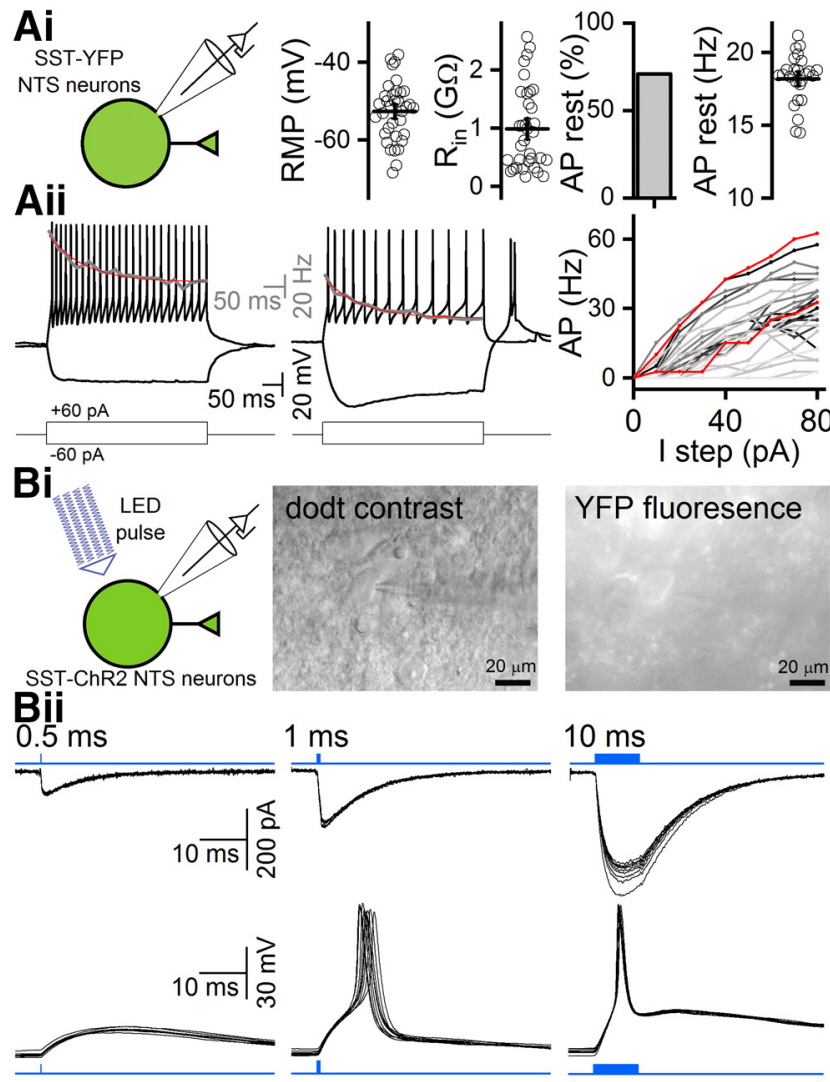

Biii
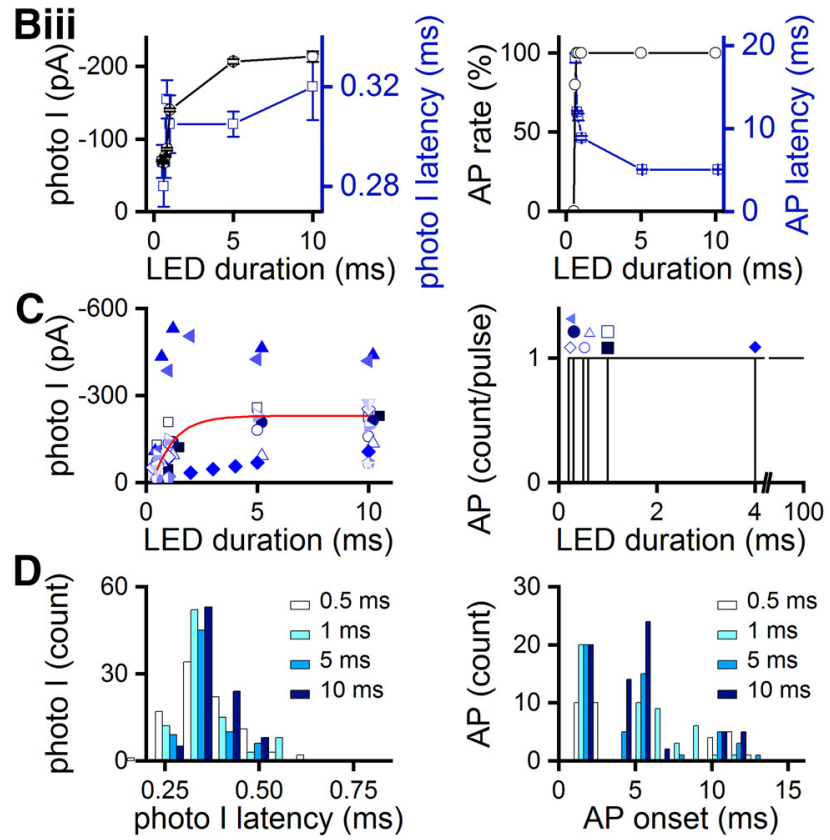

Figure 2. Accommodating spiking SST neurons are present within the NTS and SST-ChR2YFP neurons are reliably activated with LED pulses. Ai, SST-YFP NTS neurons exhibited on average relatively low resting membrane potentials (RMPs), relatively high input resistance (in $R$ ), and the majority exhibited high rates of spontaneous action potentials at rest (restAP). Aii, In response to stepped current injections, some SST neurons exhibited regular spiking which in all cases accommodated (fit in red overlay of instantaneous AP rate; gray). Across neurons, current-frequency relationships varied (example neurons to left highlighted in red $(n=30)$. Bi, Schematic showing the experimental paradigm. A representative NTS SST-ChR2-YFP-positive neuron is identified via YFP fluorescence localized to the somatic membrane and targeted for recordings (same across $\boldsymbol{B}$ ). Bii, Traces of whole-cell patch-clamp recording (10 traces are overlaid in each panel) from one representative NTSSST-ChR2-YFP-positive neuron (same across $\boldsymbol{B}$ ), demonstrating that LED pulses (blue traces with LED timing shown by solid blocks) evoked excitatory photocurrents (downward deflections in top graphs) and excitatory photopotentials
30 iterative events per neuron. Similarly, ST-evoked PSC amplitudes were based on $>20$ "successful" iterative evoked events for first PSC in a train $\left(\mathrm{PSC}_{1}\right.$ ). Failure rates (no PSC after stimulus) were calculated from a minimum of 10 light pulses or $>20$ ST test shocks. ChR2-evoked PSC variables including latency were not normally distributed and thus were compared between groups by a Kruskal-Wallis one-way ANOVA on ranks with a Dunn's post hoc test. To measure AP throughput with ST shocks, $\mathrm{AP}_{1}$ was counted across at least 30 trials, both with and without simultaneous light pulses, and compared by paired $t$ test. All spontaneous synaptic events were detected and analyzed from digitized waveforms using an off-line program (MiniAnalysis, Synaptosoft). Events smaller than the threshold detection of five times the root mean square of noise and those with multiple peaks were excluded from amplitude and decay analysis. The latter were used for determination of frequency rates. Decay-time constants were acquired by fitting a single exponential between the 10 and $90 \%$ peak amplitude portion of the current relaxation. All statistical tests were performed with SigmaStat 3.1 (Systat Software). All data are reported as mean $\pm \mathrm{SEM} ; p<0.05$ was defined as statistically significant.

\section{Results}

\section{Somatostatin neurons constitute $30 \%$ of GAD67-positive neurons within the NTS}

We examined the distribution and neurochemistry of SST neurons in the NTS of Sprague-Dawley rats using RNAscope to visualize SST, GAD67, VGAT, and vGlut mRNA-expressing cells. Across three rostrocaudal levels of the NTS, SST mRNAexpressing neurons were particularly clustered in the parasolitary, dorsomedial, and central subnuclei of the NTS (Fig. 1). Within the NTS, $65 \pm 3 \%$ of SST-positive neurons also expressed GAD67 and VGAT, and all GAD67 neurons coexpressed VGAT. The data indicate that most SST neurons can be classed as inhibitory interneurons. These were particularly clustered within the parasolitary nucleus region (Fig. 1). Of GAD67-positive neurons, $30 \pm 1 \%$ expressed SST. Thus SST NTS interneurons are a subgroup of the inhibitory network within the NTS. Both GAD67and SST-mRNA-expressing neurons were more numerous in the rostral NTS sections. The remaining cohort of SST neurons expressed vGlut $(35 \pm 3 \%)$ and were clustered within the central subnuclei of the NTS (Fig. 1). We also assessed YFP expression as an indicator of SST expression in the NTS across three SSTChR2-YFP mice using immunohistochemistry. The distribution of SST somata and fibers was very dense throughout all levels of the NTS. The ChR2-YFP construct is membrane-bound and this made it difficult to differentiate cell bodies from axons/terminals. We did observe NTS neurons with YFP in their somal mem-

\footnotetext{
to initiate APs (upward deflection in bottom graphs). Biii, The graphs are data from the same neuron showing the relationship between activation of ChR2 with increasing LED pulse duration and amplitude and latency from LED pulse onset to EPSC onsets [left; photocurrents (photo I)] and induced AP rate and latency to AP initiation (right). Photocurrent amplitude increased between 1 and $10 \mathrm{~ms}$ but photocurrent onset latencies were consistent across different pulse durations. AP initiation was reliable with pulse durations between 2 and $10 \mathrm{~ms}$ pulse with onset latency becoming more consistent and shorter with $5-10 \mathrm{~ms}$ pulse durations. These data are based on 10 trails at each pulse duration from the same neuron across $\boldsymbol{B}$. Data shown are mean \pm SEM. $C$, Graphs showing the relationship between duration of the LED pulse and resulting photocurrents in individual neurons (left; each symbol represents a different cell) and the threshold and number of APs elicited by LED pulses (right; $n=13$ neurons). Absolute photocurrent amplitudes varied across cells, yet APs were initiated $<4$ ms duration in these same neurons (symbols correspond between the left and right graphs $=8$ ). Importantly, LED pulses up to $100 \mathrm{~ms}$ never initiated multiple action potentials per pulse. $\boldsymbol{D}$, Histograms showing photocurrent count related to photocurrent latency (left) and AP count to AP onset (right). Photocurrent onset latencies were consistent regardless of pulse duration ( $n=9$ cells) yet AP initiation was variable across these neurons regardless of LED pulse duration ( $n=8$ cells).
} 
$\mathbf{A i}$
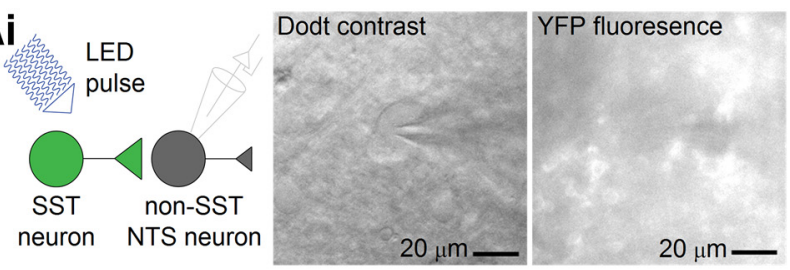

Aii

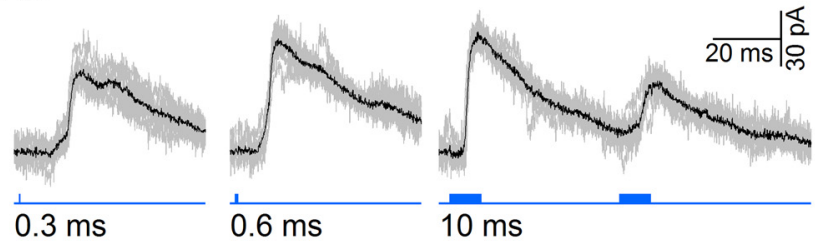

Aiii

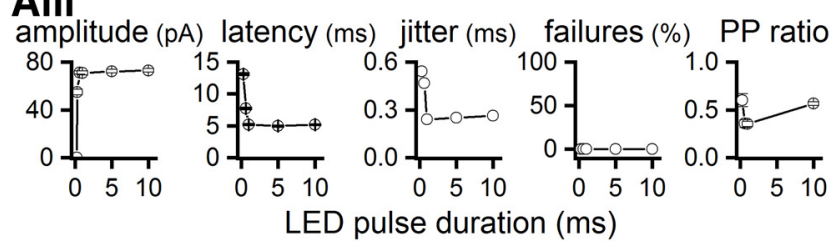

B
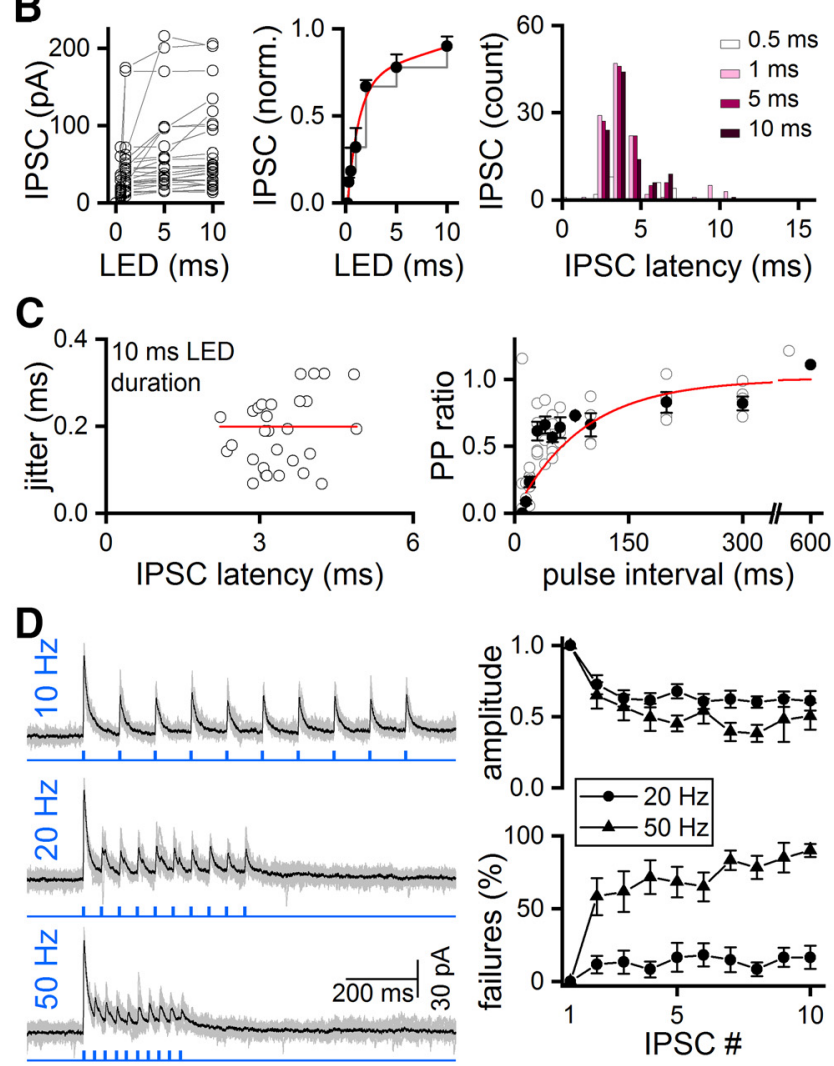

Figure 3. Non-somatostatin (SST) NTS neurons are inhibited by SST-ChR2-YFP inputs. Ai, SST-negative NTS neurons did not exhibit a photocurrent in response to LED activation of ChR2. When examined they did not express YFP (same neuron across $A$ ) and the diagram illustrating this paradigm is shown. Aii, Traces (10 traces overlaid in gray, average in black) of a whole-cell recording from a non-SST neuron in the NTS show that LED pulses (blue line with blocked area representing (hR2 on) evoked IPSCS (upward deflections) that increased in amplitude with longer LED pulse durations. Paired LED pulses (50 ms interval) induced depression in IPSC amplitude. Aiiii, For the same neuron, graphs showing the relationship between LED pulse duration and IPSC amplitude, latency to onset, variation in onset latency (jitter), failure to evoke IPSC and paired-pulse ratio (PP; mean of 10 trials). For this neuron all evoked IPSC characteristics changed with pulse durations up to $2 \mathrm{~ms}$ before plateauing, with the exception of failure rate ( $0 \%$ throughout). $\boldsymbol{B}$, Graphs showing the population data for the response of SST-negative NTS neurons to increasing pulse duration. Pulse duration (LED) is graphed against raw and normalized IPSC amplitude. The latency for initiation of IPSC is also graphed against the number of branes and those without (see Figs. 2, 3). Thus SST-expressing neurons in the NTS are mostly inhibitory and represent up to one-third of the local inhibitory population in rodents, and SST fibers are observed densely throughout the NTS.

\section{Somatostatin neurons exhibit interneuron like properties observed in other regions of the CNS}

Using a SST-YFP mouse line we were able to readily identify SST neurons within the NTS, and performed a basic electrophysiological characterization via whole-cell recordings. We sampled neurons across the medial NTS (see Fig. 8E) and their input resistance was $1059 \pm 125 \mathrm{M} \Omega$ with a resting membrane potential of $-53 \pm 1 \mathrm{mV}(n=35)$. A majority spontaneously fired APs at these resting potentials (71\%), at a rate of $18.2 \pm 0.3 \mathrm{~Hz}(14.5-$ $21.1 \mathrm{~Hz}$ range, $n=25$; Fig. $2 A i$ ). These APs exhibited a half-width of $1.77 \pm 0.50 \mathrm{~ms}$. The rheobase across all SST-YFP neurons was $18 \pm 2 \mathrm{pA}$. During current-frequency protocols we observed accommodating spiking in all recorded neurons and their peak frequency rate ranged from 3 to $63 \mathrm{~Hz}$ across cells (Fig. 2Aii). No other firing pattern was recorded (Petilla Interneuron Nomenclature Group, 2008). The AP firing accommodated in all neurons at a ratio of $0.44 \pm 0.11(n=30)$. We also observed the presence of $I_{\mathrm{h}}$ in 11 of 30 neurons with a slope of $0.23 \pm 0.03$ $\mathrm{mV} / \mathrm{pA}$ and post-inhibitory rebound spiking in 9 of 30 neurons (Fig. 2Aii). Across SST-YFP neurons there were no correlations or associations of these characteristics between cells.

\section{LED pulses reliably evoke single APs in NTS SST neurons}

We imaged YFP within in vitro slices to identify SST-ChR2-YFP neurons and to characterize the activation of these cells via ChR2 (Fig. 2Bi). These SST NTS neurons exhibited a resting membrane potential of $-55 \pm 3 \mathrm{mV}$ (range -42 to $-72 \mathrm{mV}, n=8$ ) with half exhibiting spontaneous AP firing $(2.3 \pm 1.2 \mathrm{~Hz}$, range $0.03-4.7$ $\mathrm{Hz}, n=4)$. ChR2-mediated photocurrents were studied by varying the duration of light pulses to define the assay parameters (Fig. 2Bii). These responses, defined in this study as photocurrents, are the response to activation of $\mathrm{ChR} 2$ expressed by the recorded neuron. As in the example neuron in Figure 2Bii, LED pulses evoked consistent, rapid onset excitatory photocurrents and photopotentials. LED pulses $<1 \mathrm{~ms}$ evoked photopotentials that did not reach threshold. LED pulse durations $>1 \mathrm{~ms}$ evoked reliable APs, but their onsets became more consistent with $10 \mathrm{~ms}$ pulses (Fig. 2Bii). Photocurrents reached maximal magnitude and consistent onset latency with pulses $>5 \mathrm{~ms}$. The initiation of APs in the same neuron was $100 \%$ reliable with pulses $>1 \mathrm{~ms}$ duration. As LED pulse duration increased from $1-10 \mathrm{~ms}$ we also noted AP initiation occurred sooner and more consistently (Fig. 2Biii). Across SST neurons the photocurrent magnitude varied

$\leftarrow$

IPSCS (count). ChR2-evoked IPSCs increased in amplitude with increasing pulse durations, individual data (left), normalized (middle; $n=28$, exponential function; $R^{2}=0.99$ ). The onset latencies of ChR2-evoked IPSCs were more variable at the shortest LED pulse durations tested across the group (right). C, Graphs showing the relationship between the variability of onset latency (jitter) and IPSC latency indicated ChR2-evoked IPSC synaptic jitter exhibited no relationship to onset latencies $\left(R^{2}=0.05\right.$, left). Pulse $(10 \mathrm{~ms})$ interval and PP (right) for all neurons tested. Paired-pulse ratios remained $<1.0$ (frequency-dependent depression) until the pulse interval was $>300 \mathrm{~ms}$. $\boldsymbol{D}$, Traces showing the response of a non-SST NTS neuron to multiple LED pulses (blue trace) at different frequencies $(10-50 \mathrm{~Hz}$; left; 10 traces overlaid in gray, average in black). The group data ( $n=6$ neurons) for the response to 20 and $50 \mathrm{~Hz}$ relating IPSC number to failure rate and IPSC amplitude is shown at the right. All stimulation frequencies depressed successive IPSC amplitudes relative to IPSC 1 . Where applicable all data are presented as mean \pm SEM 
greatly between neurons with $10 \mathrm{~ms}$ pulses, from 63 to $418 \mathrm{pA}$ (211 $\pm 32 \mathrm{pA}, n=13$; Fig. $2 C$, left). Because cell capacitance was similar across neurons ( $8.68 \pm 3.36 \mathrm{M} \Omega, n=13)$, this likely reflects a difference in ChR2 expression between neurons. Consequently in these same neurons AP initiation first occurred at different pulse durations, all $<4 \mathrm{~ms}$ (pulse threshold $=1.5 \pm 0.6$ $\mathrm{ms}, n=9)$. Of note, is that once AP threshold had been met in a SST neuron, each LED pulse evoked no more than one AP per pulse regardless of pulse duration up to $100 \mathrm{~ms}$ (Fig. 2C, right). We quantified the temporal relationship of $\mathrm{ChR} 2$ and AP initiation in these SST neurons. Across neurons, the photocurrent onset was $0.39 \pm 0.008 \mathrm{~ms}(n=9,10 \mathrm{~ms}$ duration $)$ and AP initiation occurred at $4.85 \pm 0.35 \mathrm{~ms}(n=7,10 \mathrm{~ms}$ duration). Thus in this slice preparation, SST NTS neurons are not spontaneously active but are reliably activated by LED pulses.

\section{SST neurons converge at SST-negative NTS neurons}

To determine how SST input gates sensory drive within the NTS we recorded from randomly sampled, medial NTS neurons without first identifying YFP expression. All SST-negative neurons, defined as those not exhibiting a photocurrent, exhibited ChR2evoked synaptically mediated responses (Fig. $3 A$ ). The most common response exhibited in SST-negative NTS neurons was ChR2-evoked IPSCs (Fig. 3Aii). These ChR2-IPSCs were blocked by TTX ( $1 \mu \mathrm{M} ; n=3$; see Fig. $8 C$ ) indicating that they were AP dependent and thus synaptically mediated. We quantified ChR2evoked IPSC characteristics in SST-negative neurons in relation to LED duration. With increasing LED pulse durations, the IPSC amplitude increased to reach a plateau between 1 and $10 \mathrm{~ms}$. The latency of ChR2-evoked IPSC onsets decreased before plateauing from 1 to $10 \mathrm{~ms}$. At all pulse durations IPSCs never failed and in conducting paired pulses, IPSCs depressed at each LED duration tested (Fig. 3Aii,Aiii). Across all neurons similarly tested ( $n=$ 28), the IPSC amplitudes induced by $10 \mathrm{~ms}$ pulses varied from 14 to $205 \mathrm{pA},(67 \pm 4 \mathrm{pA})$. When IPSC amplitude was normalized to each neuron's own maximal current, a clear relationship between IPSC amplitude and LED duration was observed (Fig. 3B). In addition, the failure rate across this cohort of neurons remained at $0 \%$ (not shown for group). Together these characteristics may indicate multiple SST inputs converging at individual SSTnegative NTS neurons.

In comparing ChR2-evoked IPSC latencies (Fig. 3B, right) to SST AP initiation (Fig. 2C, left), we observed that evoked IPSCs were both more consistent and, on average, occurred earlier to SST neurons spiking. This indicates that LED pulses are activating both SST neurons at the soma and/or SST axons that synapse on the recorded NTS neuron. There was no relationship between the synaptic jitter of ChR2-evoked IPSCs and their latency onsets (Fig. 3C, left). We varied the interval of paired pulses and found that across neurons, the amplitude of the second IPSC was depressed when pulse intervals were $<300 \mathrm{~ms}$. This indicates ChR2evoked neurotransmitter release probability is high in these conditions (Fig. 3C, right). To assess the dynamics of neurotransmitter release from SST afferents we trialed trains of pulses at 10, 20 , and $50 \mathrm{~Hz}$. We found that the IPSC amplitude of evoked responses following the first event was depressed and failures increased with subsequent multiple pulses. Second, the higher the frequency the more frequently failures occurred (Fig. 3D). In addition, high-frequency pulse trains did not alter $V_{\mathrm{H}}$ for any period after pulses, even those delivered at $50 \mathrm{~Hz}$, suggesting no other neuromodulators that may affect conductance, such as SST for example, were being released.
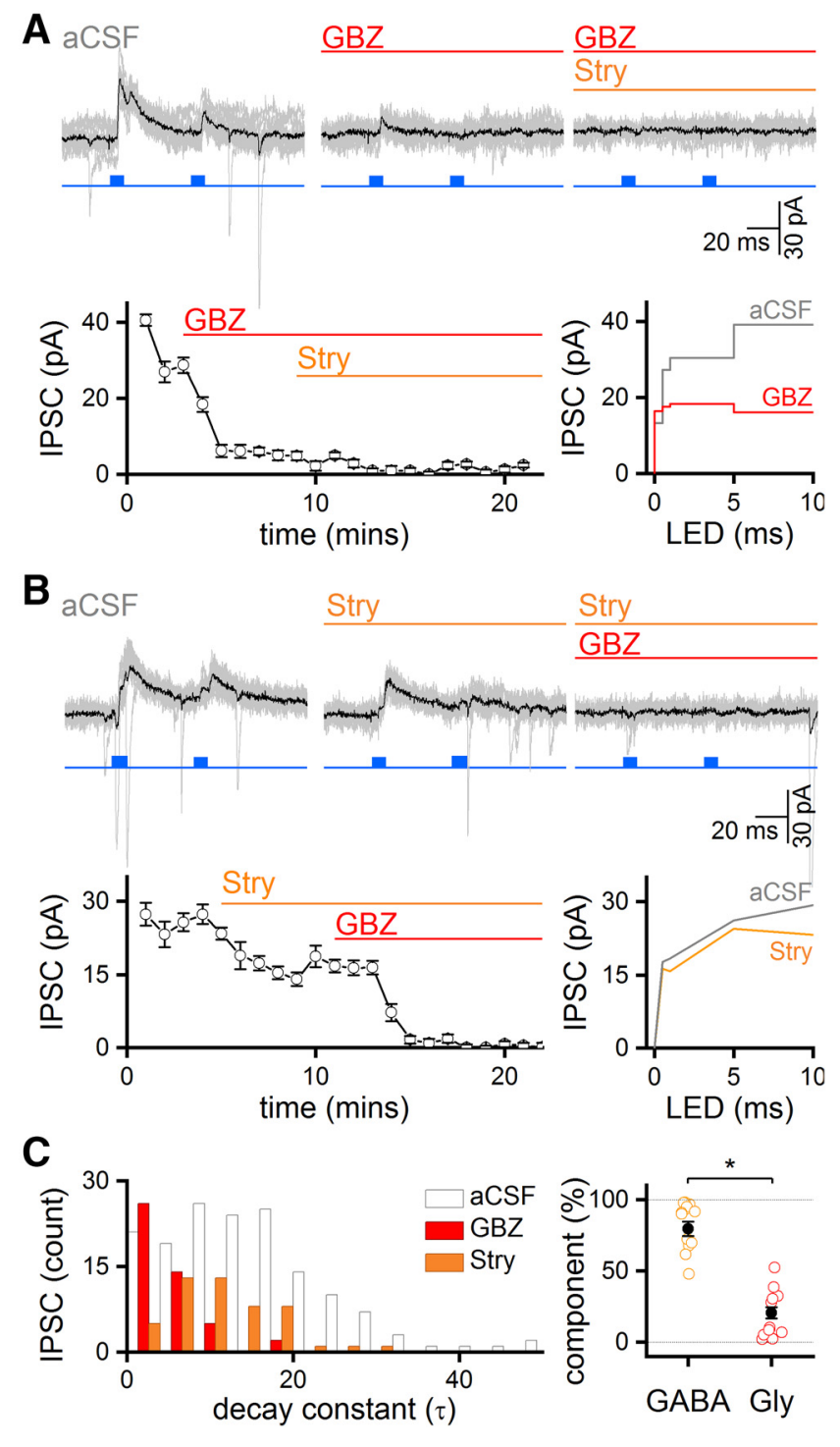

Figure 4. SST neurons utilze GABA and glycine as fast neurotransmitters in the medial NTS. $A$, Traces showing a whole-cell recordings from one representative NTS neuron (top traces; 10 traces overlaid in gray, average in black; blue traces show LED on in blocks, $10 \mathrm{~ms}$ pulses) and the associated data (bottom traces; data expressed as mean \pm SEM of 10 trials per minute). Application of the $\mathrm{GABA}_{\mathrm{A}}$ receptor antagonist, gabazine $(\mathrm{GBZ} ; 3 \mu \mathrm{m})$, substantially blocked ChR2evoked IPSCS, but not completely. The remaining IPSC was abolished by the glycinergic receptor antagonist, strychnine (Stry; $1 \mu \mathrm{M}$ ). LED-evoked IPSC thresholds and amplitude (bottom right trace), in both aCSF and GBZ, indicated recruitment profiles aligned, suggesting $\mathrm{co}^{-}$ transmission of the neurotransmitters. $\boldsymbol{B}$, Traces from another representative NTS neuron showing a similar protocol where the order of antagonist application was reversed, similar results were obtained. $C$, Histograms relating the number of IPSCS induced by LED stimulation to the decay constant in the absence (aCSF) and presence of $\mathrm{GABA}_{A}$ or glycine receptor antagonists ( $n=9$ neurons). Glycinergic IPSCS (GBZ condition; red) exhibited faster dynamics than GABAergic IPSCS (orange) to the same neurons. Individual data (right graph; $n=16$ neurons) from SST-negative NTS neurons demonstrates that GABA was responsible for a greater proportion of the IPSC transfer $(80.9 \pm 5.0 \%)$ compared with glycine $\left(9.7 \pm 4.8 \%\right.$; ${ }^{*} Z=-3.361, p \leq$ 0.001 , Wilcoxon signed rank test), but this varied greatly between cells.

\section{SST neurons use both GABA and glycine to gate second-order} neurons in the NTS

To determine the neurotransmitters generating SST-mediated IPSCs we used pharmacological agents to block responses (Fig. 4). It was expected that SST neurons would be GABA-ergic, however bath application of $\mathrm{GABA}_{\mathrm{A}}$ receptor antagonist, gabazine (SR-95531, $3 \mu \mathrm{M}$; Tocris Bioscience) completely ( $n=3$ of 16 ) or 


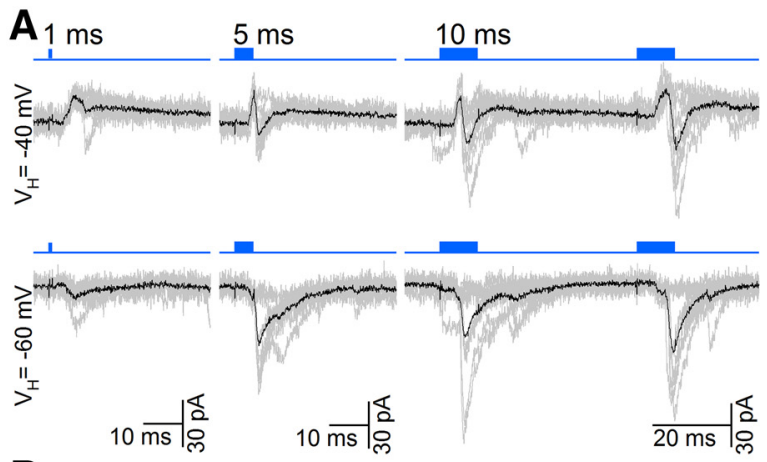

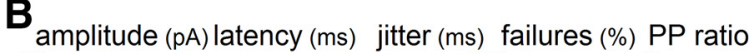
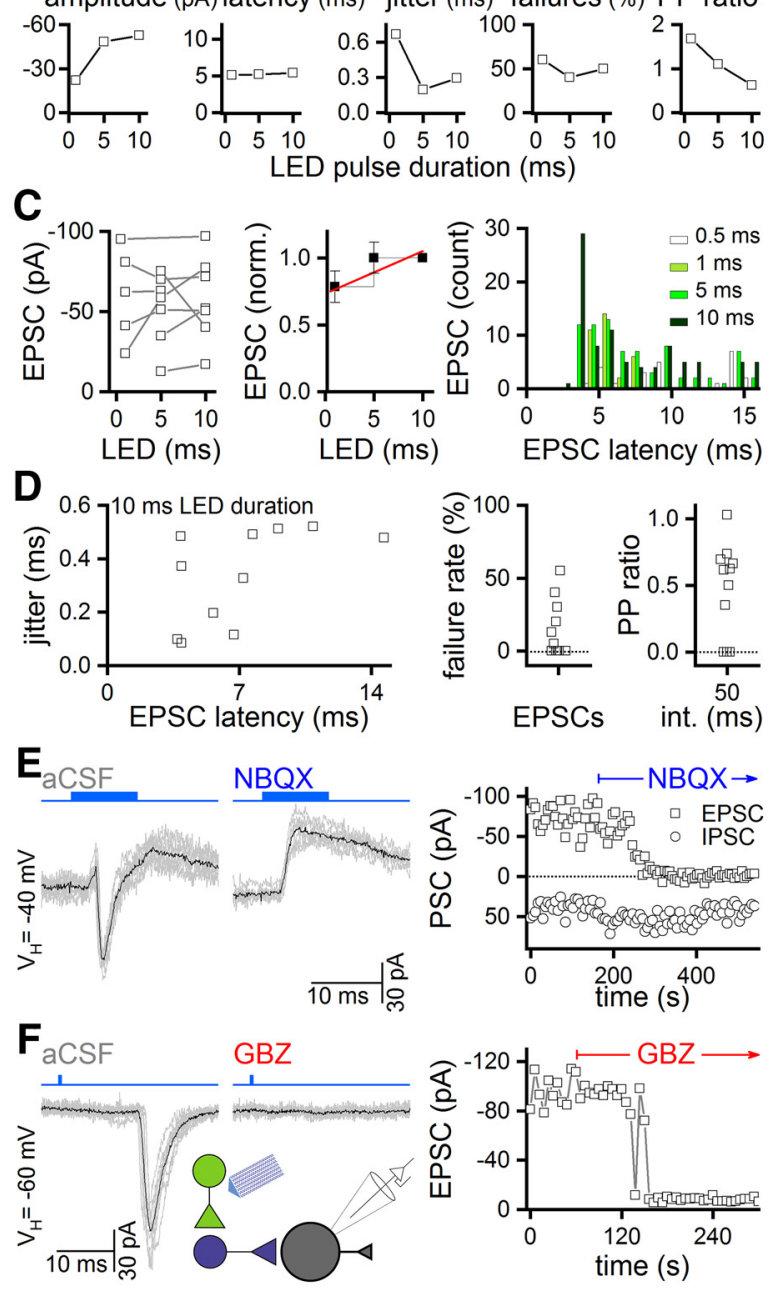

Figure 5. SST neurons evoke excitatory drive via post-inhibitory rebound $A$, Traces from a whole-cell recording of a non-SST NTS neuron (10 traces overlaid in gray, mean trace in black; blue trace showing LED pulse durations). EPSCs $\left(V_{H}=-60 \mathrm{mV}\right)$ were differentiated from IPSCs $\left(V_{H}=-40 \mathrm{mV}\right)$ by an inward (downward deflection) fast decaying current at $V_{H}=-40 \mathrm{mV}$ and became more prominent at $-60 \mathrm{mV}$ (close to $\mathrm{E}_{\mathrm{Cl}}$ ). $\boldsymbol{B}$, Graphs for the representative neuron shown in $\boldsymbol{A}$, showing the effect of increasing LED pulse duration on EPSC amplitude, latency, variability in onset (jitter), failure rate and paired-pulse ratio (PP). The EPSC amplitude increased with LED duration, latency was constant yet failure rate was high at each LED duration tested in contrast to IPSCS (Fig. 3). C, Graphs showing group data from NTS neurons $(n=11)$ with only EPSCS, and those where EPSCs were isolated from IPSC/EPSC complexes. Individual raw data (left), and normalized group data (middle; mean \pm SEM) are shown. Across neurons, ChR2-evoked EPSCs remained constant or increased in amplitude with increasing LED pulse durations. The onset latencies of ChR2-evoked EPSCs (right graph) were variable regardless of LED pulse durations and arrived significantly later than ChR2-evoked IPSCS (compare to Fig. 3B; $p \leq 0.001$, one-way ANOVA on ranks). $\boldsymbol{D}$, No relationship between EPSC latency and onset latency variability (jitter; left) was observed. In contrast to $0 \%$ failure rate for IPSCS (Fig. 3), EPSC failure rates were variable across neurons (middle). When the PP could be measured, it was substantially, but incompletely blocked IPSCs ( $n=13$ of 16 ). Subsequent bath application of the glycine receptor antagonist, strychnine ( $1 \mu \mathrm{M}$; Sigma) abolished the remaining IPSC component (Fig. 4A). To determine whether GABA and glycine release could be differentially recruited we varied the LED pulse duration to see whether evoked IPSCs would be altered after the addition of the blockers. In all cases, the GABA or glycine alone IPSCs exhibited the same LED duration threshold, to aCSF control trials. One possible implication of these data is that these neurotransmitters are co-released (Fig. $4 A, B$, bottom right). ChR2evoked IPSCs were fitted for single exponential decay constants (90-10\%) and pharmacologically-isolated glycine currents decayed 2.07 times faster $(5.35 \pm 1.00, n=48$ trials, $\mathrm{H}=53.29$, p $=<0.001$, df $=2$; Kruskal-Wallis one-way ANOVA on ranks). than isolated GABA currents $(11.1 \pm 0.92, n=49$ trials; Fig. $4 C$, left), confirming their separate kinetics. Analysis of the peak IPSC amplitude (mean of 10 trials per condition) in aCSF, gabazine, and strychnine established that GABA was responsible for the majority component of IPSCs, contributing $79 \pm 5 \%$ compared with glycine's $21 \pm 5 \%$ for each event ( $n=16$; Fig. $4 C$, right).

\section{SST neuron activation evokes EPSCs via polysynaptic pathways within NTS}

In several SST-negative neurons ChR2 activation evoked mixed IPSC/EPSC complexes or EPSCs alone (Fig. 5). Evoked EPSCs were differentiated from evoked IPSCs by varying $V_{\mathrm{H}}$ of the recorded neurons. Chloride-mediated PSCs are outward and $\mathrm{Na}^{+}$/ $\mathrm{Ca}^{2+}$-mediated PSCs are inward at $-40 \mathrm{mV}$ using a $10 \mathrm{mM} \mathrm{Cl}^{-}$ internal solution. In the same neurons, the driving force for $\mathrm{Cl}^{-}$ is minimal at $-60 \mathrm{mV}$ and this effectively isolates the remaining evoked EPSCs (Fig. 5A). ChR2-evoked EPSCs occurred in 4\%, whereas mixed IPSC/EPSC complexes occurred in 15\% of SSTnegative neurons tested $(n=72)$. Given up to one-third of NTS SST neurons coexpress vGlut (Fig. 1D), these rates of excitatory input were surprisingly low. We performed detailed analysis of ChR2-evoked EPSC amplitude, latency, jitter, failure rate, and paired-pulse ratio (Fig. 5B). In neurons exhibiting ChR2-evoked EPSCs, event amplitudes remained constant with increasing LED durations (Fig. 5C, left) indicating recruitment of a single excitatory input to the recorded neurons. We compared the onset latency of ChR2-IPSCs (at $V_{\mathrm{H}}=-40 \mathrm{mV}$; Fig. $3 B$ ) to ChR2EPCSs (at $V_{\mathrm{H}}=-60 \mathrm{mV}$; Fig. $5 \mathrm{C}$ ) and surprisingly, ChR2evoked EPSCs had a longer latency to onset compared with evoked IPSCs (compare Figs. $5 C$, right, $3 B$, right; $\mathrm{H}=210.55, p \leq$ 0.001 , df $=1$; Kruskal-Wallis one-way ANOVA on ranks). Within neurons receiving mixed input, we noted complex PSCs exhibited outward (inhibitory) followed by inward (excitatory) components in $V_{\mathrm{H}}-40 \mathrm{mV}$ traces (Fig. $5 \mathrm{~A}, E$ ). The jitter of EPSCs was higher compared with evoked IPSCs and the failure rate ranged up to $50 \%$ compared with $0 \pm 0 \%$ for evoked IPSCs. It is interesting to note that the paired-pulse ratio was $<1.0$ for

\section{$\longleftarrow$}

generally $<1.0$ at a $50 \mathrm{~ms}$ pulse interval (right). $\boldsymbol{E}$, Traces of a whole-cell recording from one SST-negative NTS neuron (10 traces overlaid in gray, mean trace in black; blue trace showing LED activation) showing evoked EPSCs in aCSF and following blockade of AMPA receptors (NBQX, $20 \mu \mathrm{m}$ ). The effect of the antagonist on the amplitude of PSCs is shown (right) with NBQX blocking EPSCS and not affecting IPSCS to this neuron. $\boldsymbol{F}$, Traces from another SSTnegative NTS neuron (10 traces overlaid in gray, mean trace in black; blue trace showing LED activation) that only received EPSCs in response to ChR2 activation. Application of the $G A B A_{A}$ receptor antagonist, gabazine (GBZ; $3 \mu \mathrm{M})$, blocked LED-evoked EPSCs and decreased EPSC amplitude to zero (right). The diagram indicates a polysynaptic pathway with either postinhibitory rebound or disinhibition mechanisms being responsible. 
most events (Fig. 5D). Combined, the data indicates that ChR2evoked EPSCs are not likely to be directly synapsing at recorded NTS neurons. This was confirmed pharmacologically in neurons that received mixed PSC complexes. Evoked EPSCs were blocked by the AMPA receptor antagonist, NBQX, which did not block ChR2-evoked IPSCs (Fig. 5E). The $\mathrm{GABA}_{\mathrm{A}}$ receptor antagonist, gabazine (SR95531), blocked IPSCs as expected, but also blocked ChR2-evoked EPSCs. Thus the excitatory input to recorded NTS neurons required SST inhibitory neuron activation and is presumably the response to either disinhibition or rebound excitation in excitatory neurons.

\section{SST neurons impact viscerosensory glutamatergic} transmission to second-order NTS neurons

Electrical ST stimulation evokes glutamate release from viscerosensory inputs that is subject to $\mathrm{GABA}_{\mathrm{B}}$ modulation (Fawley et al., 2011). To determine whether SST neurons provide a presynaptic inhibitory input to viscerosensory afferents, we first assessed whether the spontaneous EPSC frequency was altered in the presence of $\mathrm{GABA}_{\mathrm{A}}$ and glycine receptor antagonists (gabazine, $3 \mu \mathrm{M}$; strychnine, $1 \mu \mathrm{M})$. Spontaneous EPSCs in second-order NTS neurons are, in part, because of glutamate release from ST primary afferents (Peters et al., 2010, 2011). Under this inhibitory ion channel blockade we examined the effect of several LED pulse routines on spontaneous EPSC frequency, varying pulse number $(1,2,5,10)$ and frequency $(2,20,50 \mathrm{~Hz})$ of $10 \mathrm{~ms}$ LED pulses (Fig. $6 A)$. The sEPSC frequency did not change in any condition tested in any neurons $(n=4$; Fig. $6 B)$. In another series of experiments we tested whether SST input alters ST-evoked EPSCs in secondorder NTS neurons. We established that recorded neurons received both SST (ChR2)-evoked IPSCs and low jitter ST-evoked EPSCs and then bath applied $\mathrm{GABA}_{\mathrm{A}}$ and glycine receptor antagonists as above (Fig. 6C). Here too we trialed the pulse routines varying pulse number $(1,2,5,10)$ and frequency $(2,20,50 \mathrm{~Hz})$ of $10 \mathrm{~ms}$ LED pulses, and timed these to precede ST-evoked EPSCs, to assess whether ST-evoked glutamate release would be impacted (Fig. $6 C$ ). Across neurons $(n=5)$, ST-evoked EPSCs, shocks $1-5$, tended to exhibit smaller amplitudes compared with control responses, but this was not statistically significant (Fig. $6 D)$. We conclude that SST neurons do not terminate presynaptically on visceral sensory afferents to mediate $\mathrm{GABA}_{\mathrm{B}}$ receptor modulation of transmitter release.

\section{SST neurons gate viscerosensory signals at second-order NTS neurons}

We next determined the impact of coincident SST input on viscerosensory input, determined by electrical stimulation of the ST (Fig. 7). We characterized cells in this protocol by first showing that a series of ST shocks alone reliably initiated APs in secondorder NTS neurons (Fig. 7B). LED pulses alone reliably evoked IPSPs in neurons, even with small driving potentials $\left(\mathrm{E}_{\mathrm{Cl}-}=\right.$ $-68.8 \mathrm{mV}$ ), at resting membrane potentials (Fig. $7 B$ ). We found that SST-mediated hyperpolarization blocked spontaneous AP firing in these NTS neurons and delayed the onset of AP initiation in response to depolarizing current injections (data not shown). When the delivery of ST electric shocks was timed to coincide with LED pulses in second-order NTS neurons, we observed that ST-initiated APs were prevented, as ST-evoked EPSPs did not reach AP threshold (Fig. $7 B$ ). Across neurons we trialed ST shocks, with and without LED pulses, consecutively for a total of 60 trials each (Fig. 7C). Across the group AP throughput varied from 83 to $100 \%$ with ST shocks alone. The AP throughput decreased to between 0 and $63 \%$ in these same neurons with SST-
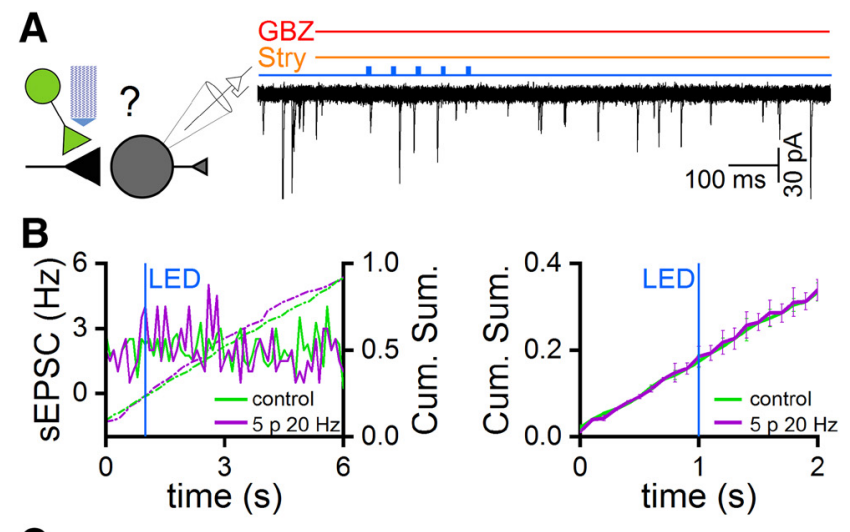

C

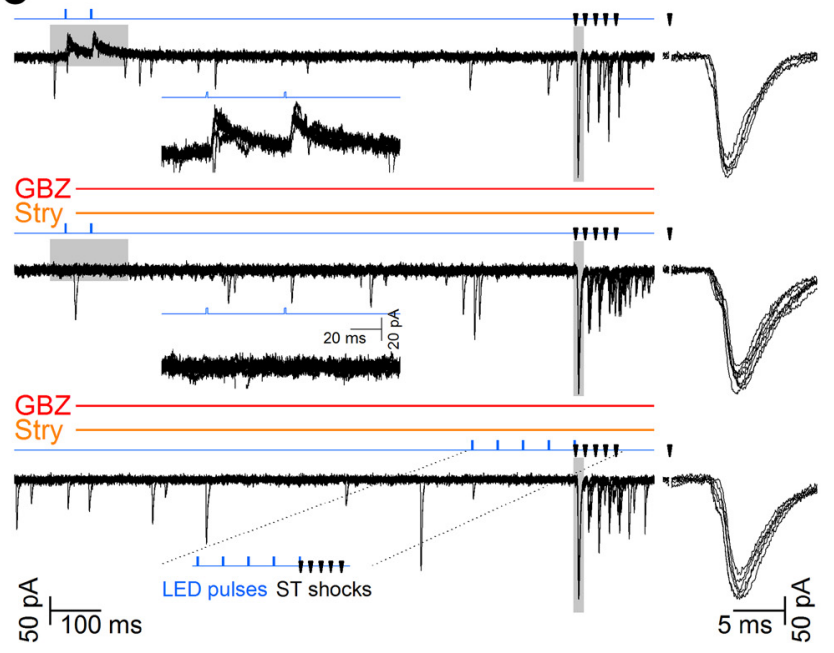

D
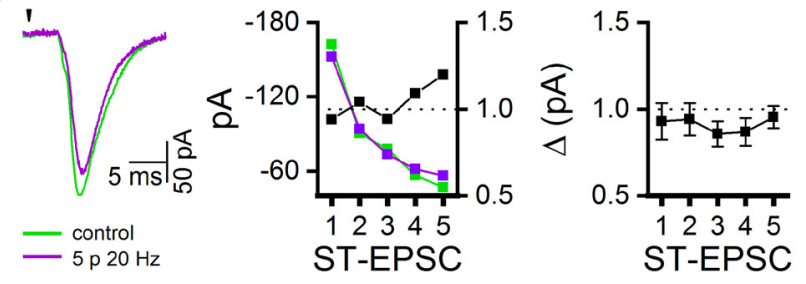

Figure 6. SST neurons do not modulate glutamate release presynaptically at SST-negative NTS neurons. $\boldsymbol{A}$, Traces showing spontaneous EPSCS (sEPSC) recorded from an NTS neurons in the presence of $\mathrm{GABA}_{\mathrm{A}}$ and glycine receptor antagonists; gabazine $(\mathrm{GBZ} ; 3 \mu \mathrm{m})$ and strychnine (Stry; $1 \mu \mathrm{M}$ ) as well as ChR2-evoked activity (blue traces indicates LED activation during the blocks). $\boldsymbol{B}$, In the neuron shown in $\boldsymbol{A}$, the sEPSC frequency remained constant during and after repeated ChR2 pulses as confirmed by cumulative summation analysis (left). Similar results were obtained across all neurons examined ( $n=4$; right). C, Traces showing whole-cell recordings of a SST-negative NTS neuron before, and during, bath application of the $G_{A B A}$ receptor antagonist GBZ $(3 \mu \mathrm{M})$ and glycinergic receptor antagonist Stry $(1 \mu \mathrm{M})$. LED pulses (10 ms pulses at $20 \mathrm{~Hz}$ ) to activate (hR2 were delivered at the times shown in the blue trace. Stimulation of viscerosensory input via the ST ( 5 shocks at $50 \mathrm{~Hz}$ ) occurred at the time of the inverted black triangles. The periods highlighted with gray boxes are shown on in the adjacent higher resolution insets (10 traces overlaid). Blockade of inhibitory ionotropic receptors abolished IPSCs in response to LED activation. Precisely timed LED stimulation just before ST shocks in the presence of ionotropic receptor blockade did not alter the response to ST input. $\boldsymbol{D}$, Average trace of the first EPSC in response to ST stimulation in the neuron from $\boldsymbol{C}$ showed little difference in EPSC1. Graphs of EPSC amplitude across all five ST shocks from five different cells illustrate that stimulation of SST inputs in the NTS slice did not alter ST-evoked EPSCs $\left(F_{(4,1)}=3.305, p=\right.$ 0.143; two-way ANOVA with repeated measures; data are mean \pm SEM).

mediated IPSPs. On average ST throughput decreased from $90 \pm$ 4 to $23 \pm 10 \%$ (Fig. 7C). Thus, SST input can gate viscerosensory input to second-order NTS neurons, effectively isolating these neurons from their sensory input. 
Extensive SST-mediated gating throughout the NTS

Given this powerful ability of SST inputs to gate viscerosensory input, we attempted to understand the likely impact on the NTS population. As summarized in Figure 8, we observed that all recorded non-SST, NTS neurons received an SST input. We first determined whether each recorded NTS neuron received primary afferent input (ST-evoked EPSCs; $50 \mathrm{~Hz}$, 5 shocks). Those neurons that exhibited ST-EPSCs, with jitter $<200 \mu$ s for EPSC $_{1}$, and those that exhibited frequencydependent depression across EPSC $_{1-5}$ were defined as second-order neurons (Fig. 8A). Responses with synaptic jitter $>200 \mu \mathrm{s}$ for $\mathrm{EPSC}_{1}$ and no frequencydependent depression across EPSC $_{1-5}$ were defined as higher-order NTS neurons (Fig. 8B). Second, to determine whether neurons were SST expressing and/or received SST input, we analyzed ChR2-evoked PSCs. We classified NTS neurons exhibiting reproducible inward currents, with onsets that were synchronized with the onset of LED pulses (i.e., photocurrents), as SST expressing (Fig. $8 C$, left). These PSCs were not affected by TTX. Neurons that exhibited ChR2evoked PSCs with variable onsets were classified as receiving SST input (Fig. 8C, right). When tested, these inputs were blocked by TTX indicating they were synaptically mediated. In some cases a combination of both photocurrents- and ChR2 evoked-PSCs were exhibited, defining SST NTS neurons that also received an SST input (Fig. $8 C$, middle).

We observed a relatively low density of SST neurons within the NTS, in concor-

dance with our RNAscope studies (Fig. 1), yet a widespread influence of SST inputs. When we randomly recorded NTS neurons without prior visualization of YFP, in SST-ChR2-YFP mice, most neurons recorded were SST-negative $(n=72)$, and only two SST-positive neurons were sampled this way (74 instances). In considering neurons we recorded from in SST-ChR2-YFP mice, including those reported in Figure 2 identified by direct visualization of YFP, we found that every SST-negative neuron $(n=72)$ received ChR2-evoked PSCs (72/72; 58 receiving IPSCs, 11 receiving IPSCs and EPSCs, and 3 receiving EPSCs; Fig. $8 D$ bottom right). In SST-positive neurons, 4/16 also received input from other SST neurons (Fig. 8D, bottom left). Thus, SST inputs terminate extensively within the NTS to gate the vast majority of neurons.

Across SST-YFP and SST-ChR2-YFP mouse lines we defined recorded neurons in relation to primary afferent input. Electrical stimulation of the ST in 90 neurons, 42 SST-positive and 48 SSTnegative neurons, characterized the majority of both SSTpositive (24/42) and SST-negative neurons (30/48) as second order (Fig. $8 D$, top). The remaining neurons were either classified as higher order (SST-positive: 11/42, SST-negative: 15/48) or as receiving no viscerosensory input (Fig. $8 D$, top). Thus, most SST neurons are driven directly by primary afferent input, indicating a prominent feedforward inhibition arrangement within the NTS. The relative locations of patched neurons within the NTS were mapped with no clear topographical delineation for second or higher order, or SST-positive and -negative neurons observed in this horizontal plane (Fig. 8E).

\section{Discussion}

The NTS occupies a critical position as the first synapse for viscerosensory information entering the brain. Any modulatory factor or mechanism operating at the level of the NTS is thus very powerful as it will alter all subsequent information processing. Remarkably, every SST-negative NTS neuron we recorded from had received input from at least one SST neuron. Inhibition was mediated via both GABA and glycine. Activation of SST inputs powerfully gate viscerosensory signal throughput postsynaptically, with the potential to disengage second-order NTS neurons from their sensory input. We posit that the SST inhibitory input within the NTS is pivotal in coordinating and modulating viscerosensory integration at its initial stages.

Whereas the vagal afferent synapse in the NTS provides high reliability glutamatergic transmission (Doyle and Andresen, 

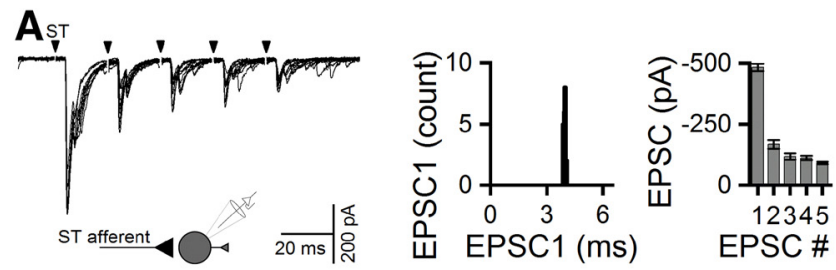

$\mathrm{B}_{\mathrm{s}}$
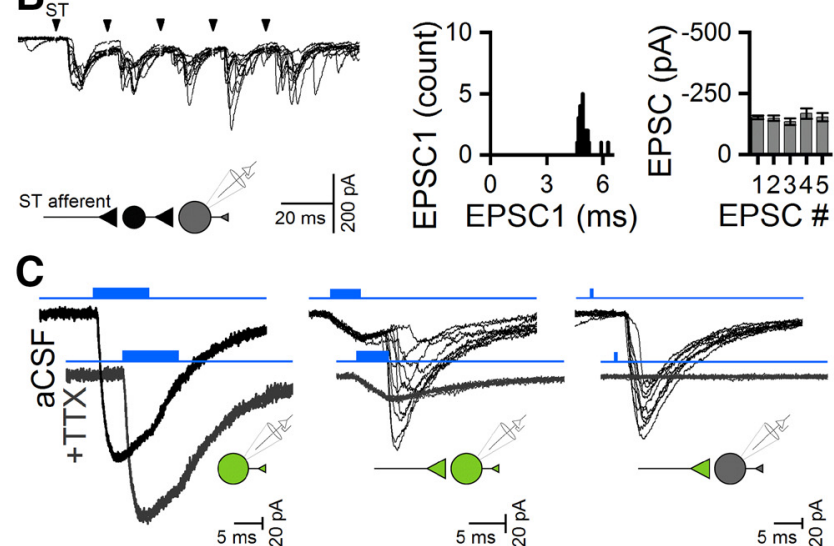

D

somatostatin +ve neurons

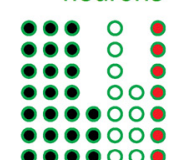
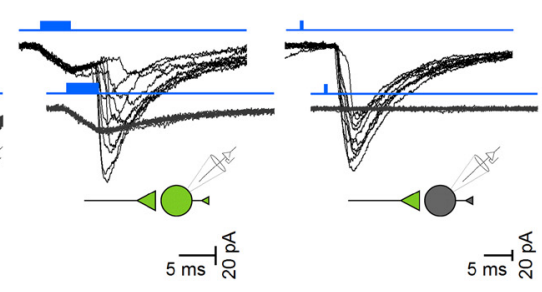

somatostatin -ve neurons

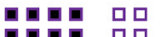

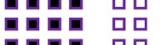

븜ำ

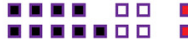

- 2nd order neuron

$\square$ higher order neuron

$\square$ no ST input

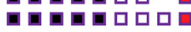

부믐ㅁㅁㅁㅁㅁㅜ

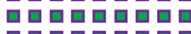

뭄ํㅁํㅁำ

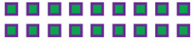

ㅁำำㅁำ

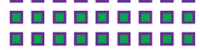

$\square$ SST input

$\square$ no SST input

000

이을

000

E

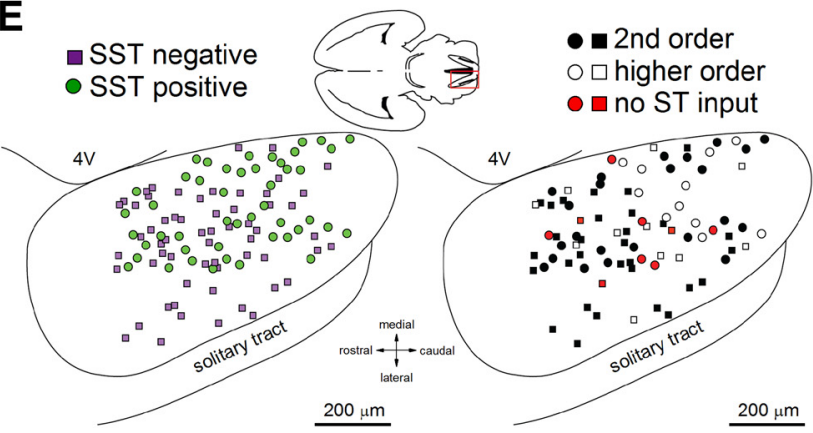

Figure 8. SST-negative NTS neurons receive somatostatin input and most somatostatinpositive neurons are second-order NTS neurons, indicating prominent feedforward inhibition. $\boldsymbol{A}$, Traces of whole-cell recording of a non-SST NTS neurons during stimulation of the ST (arrows; $50 \mathrm{~Hz}$ ) showing evoked EPSCs (10 traces overlaid). Consistent latency to onset of ST-EPSC1 (middle histogram) and frequency-dependent depression (right histogram; data are mean \pm SEM) defined second-order neurons. In this representative neuron repeated ST shocks evoked consistent EPSCs with a variability (jitter) of $51 \mu \mathrm{s}$. $\boldsymbol{B}$, Similar recordings and data analysis from another non-SST NTS neuron showing ST-EPSCS of variable onset latencies (middle; jitter $=347 \mu \mathrm{s}$ ) and negligible EPSC amplitude depression (right), defining it as higherorder neuron. C, Traces of whole-cell recordings (10 overlaid traces) from three different neurons showing ChR2-evoked responses (blocked areas in blue traces) in aCSF, or in the presence of tetrodotoxin (TTX), to block voltage-gated sodium channels. Recording conditions meant that in these traces both $\mathrm{Na}^{+} / \mathrm{Ca}^{2+}$ (excitatory) and $\mathrm{Cl}^{-}$(inhibitory) currents are inward (downward deflections). The diagrams show the circuit arrangement defined by these responses. These differentiate SST-positive neurons that do not receive an SST input, these show consistent PSC with onsets simultaneous to LED pulses (left); from SST-positive neurons, which also receive an SST input, these show consistent ChR2 PSCs followed by variable PSCS
2001), considerable modulation occurs at this first synapse. This includes actions via a diverse range of presynaptic metabotropic glutamate, $\mathrm{GABA}_{\mathrm{B}}$, and peptide receptors (Jin et al., 2004; Bailey et al., 2006; Fawley et al., 2011). Microinjections of GABA and glycine receptor agonists and antagonists into the NTS affect all reflexes tested in cats, dogs, rats, and rodents (Catelli et al., 1987; Kubo and Kihara, 1987; Herman et al., 2009; Cinelli et al., 2016), demonstrating the potent effect of these inhibitory networks within NTS. In this study we tested whether SST neurons might be a source of GABA acting at presynaptic ionotropic or metabotropic receptors on vagal afferents terminals. We did not find evidence to support this. Rather, we found extensive modulation by SST neurons at the next opportunity to gate incoming signals, at second-order NTS neurons. Where every recorded SST-negative neuron, the majority of which were second-order neurons, received an input from an SST neuron. Furthermore we observed summation of ChR2-evoked IPSCs in SST-negative neurons with increasing pulse duration, indicating that SST inputs converge onto second-order neurons. Convergence of inhibitory SST inputs allows for degrees of inhibition at second-order NTS neurons. Here we demonstrated that SST-mediated hyperpolarization competes with ST-evoked excitation of viscerosensory input to prevent second-order neurons from reaching threshold. This mechanism requires precise timing and provides one way that incoming viscerosensory signals can be coordinated. Thus SST input form part of a microcircuit gate that controls viscerosensory signal throughput. Because 70\% of GAD67 NTS neurons are SST-negative it would be desirable to define their role, in part to place the SST inhibitory network in context, and know whether it is uniquely positioned, or not, within NTS to gate viscerosensory signals.

Most SST neurons in the NTS are second-order neurons, occurring at rates similar to that observed previously in a GAD67GFP (GIN)-labeled mouse (Bailey et al., 2008). As a consequence, activation of their extensive inhibitory influence within the NTS is likely in phase with viscerosensory input. We have reported previously that stimulation of viscerosensory input evoked polysynaptic IPSCs in NTS neurons (McDougall et al., 2017) and, from the results of this study, it is likely this feedforward inhibition is mediated by SST neurons. In this study we could not determine the modality (baroreceptor or chemoreceptor, for example) of viscerosensory inputs at SST neurons, and therefore whether this integration is specific to particular sensory modalities. We also cannot determine the extent of the effect on downstream targets of second-order neurons receiving SST input. One likely efferent target is the parasympathetic motor neurons within the dorsal motor nucleus of the vagus nerve

$\leftarrow$

(middle); and SST-negative neurons receiving an SST input (right), a neuron exhibiting only IPSCs after LED pulse onsets. $\boldsymbol{D}$, Graphical representation of the properties observed across the recorded population of NTS neurons, classified as described in $\boldsymbol{A}-\boldsymbol{C}$. Across the population of NTS neurons, SST-positive (left, green outline circles) and -negative neurons (right, magenta outline squares), the majority were second order (57-63\%; black fill), a smaller proportion higher order (26-31\%; white fill) and a small proportion did not exhibit a response to ST stimulation (no input; red fill). Of NTS SST neurons only $25 \%$ received input from other SST neurons (bottom left quadrant; green filled circles). All recorded SST-negative NTS neurons received SST input (bottom right quadrant; green filled squares). $\boldsymbol{E}$, The locations of recorded neurons were mapped from photomicrographs. SST-positive (left, circles) and -negative (right, squares) neurons were distributed across the medial NTS. Similarly, synaptic order, determined by ST afferent input characteristics, was distributed relatively evenly throughout the medial NTS. 
(DMX), the majority of which are modulated by GABAergic input (Babic et al., 2011). Utilizing a similar SST-ChR2 mouse model as reported here, Lewin et al. (2016) showed that stomach-projecting DMV neurons were inhibited by activation of ChR2. They concluded this input would be from DMV interneurons expressing SST, however, the data here suggests that input from NTS SST-positive neurons would also be activated. This hypothesis has yet to be directly tested and a comprehensive map of the projections of SST-positive neurons in the NTS should be undertaken.

Using RNAscope within the rat NTS showed a sparse distribution of SST neurons throughout the nucleus and concentrated populations in the parasolitary, dorsomedial, and central subnuclei. Our RNAscope data for GAD-67 expression though the NTS aligns with previous mapping in a GAD-67-GFP mouse model (Okada et al., 2008) and single-cell expression data in the central subnucleus (Babic et al., 2015). Approximately one-third of the SST neurons we observed did not express GAD-67. We therefore colabeled for VGAT and vGlut to assess these remaining SST neurons. Surprisingly we find all remaining (non-GAD67) SST neurons expressed message for vGlut and are therefore likely excitatory. Yet our electrophysiological data indicate most SST input to non-SST neurons was inhibitory. The ChR2-evoked EPSCs we observed arrived significantly later than the evoked IPSCs and this temporal delay indicates a polysynaptic pathway was being activated. A very similar observation was made in a GAD-65-ChR2 mouse model (Chen et al., 2016), within the rostral NTS. The excitatory ChR2-evoked EPSCs we observe were also blocked by a $\mathrm{GABA}_{\mathrm{A}}$ antagonist. We conclude the evoked EPSCs might occur via disinhibition, as we observed some SSTexpressing neurons also received inhibitory SST input. Alternatively, activation of the SST input may evoke post-inhibitory rebound in excitatory neurons because this has been documented to occur in a population of NTS neurons (Paton et al., 1993, 2001; Vincent and Tell, 1999) and here in some SST NTS neurons. Given these results and the previous literature we hypothesize that NTS excitatory SST neurons project outside the nucleus and are not involved in intranuclear gating or integration like that of the inhibitory SST cohort.

The relatively low density of SST neurons that we observed in the NTS is interesting in light of the high rates of SST inputs we measured at SST-negative neurons. We observed that SSTnegative NTS neurons received multiple SST inputs. We interpret this to indicate that each SST neuron contacts multiple neurons. In the visual cortex SST neurons are similarly distributed through the cortical layers, innervate widely across other subclasses of inhibitory neurons, but not at other SST neurons (Pfeffer et al., 2013). Although this organizational structure seems to hold broadly, some SST neurons in the NTS do receive input from other SST neurons. With ubiquitous SST input to NTS neurons, this arrangement may allow for a synchronous signal to be generated from the NTS. An alternative that needs to be considered with the current experimental paradigm is that we are activating all SST-ChR2 inputs within the slice. These do not only arise from local NTS neurons. For example, GABA-ergic, SST neurons in the central amygdala project to the NTS (Saha et al., 2002) and the terminals of these neurons would be activated in our study. Thus, although we propose a strong and extensive SST interneuronal network within the NTS, our data does not rule out descending modulatory inputs.

In carrying out an electrophysiological characterization of the SST NTS neurons we observed accommodating (adapting) spiking patterns in all neurons. A portion exhibit $I_{\mathrm{h}}$, and some post-inhibitory rebound. Yet none of these factors correlated across the sample neurons to enable them to be divided into distinct groups. These are characteristics observed in SST neocortical and GABAergic cerebral cortex interneurons (Petilla Interneuron Nomenclature Group, 2008; McGarry et al., 2010). A key feature of SST GABAergic neurons in the hippocampus and neocortex is that they exhibit high spontaneous firing rates, due to intrinsic membrane conductances, even in in vitro slice preparations (Urban-Ciecko and Barth, 2016). This provides the capability to tune activity to increase or decrease firing according to need. In our study over two-thirds of SST neurons were spontaneously spiking at resting membrane potentials. Because many vagal afferents operate tonically within an operating range this would provide the drive for dynamic upregulation or downregulation of the SST network within the NTS.

Another feature of the SST input to NTS neurons is that transmission was via GABA and glycine. As shown previously for miniature IPSCs within NTS (Dufour et al., 2010) and DMV (McMenamin et al., 2016), we observed that the glycinergic currents decayed much faster than the GABAergic currents. It is well established that GABAergic neurons are found extensively throughout the NTS (Fong et al., 2005; Bailey et al., 2008) and that all NTS neurons exhibit GABA-mediated IPSCs (McDougall and Andresen, 2012). Yet the glycinergic system is more nuanced. Previous reports indicate a strict anatomical separation with glycine receptor-mediated miniature IPSCs recorded in the lateral but not medial NTS (Dufour et al., 2010). In previous in vitro slice studies, IPSCs evoked by local intranuclear electrical stimulation were fully blocked by a $\mathrm{GABA}_{\mathrm{A}}$ receptor antagonist (McDougall and Andresen, 2012; Ostrowski et al., 2014). In the current study we also did not observe any spontaneous IPSCs in the presence of $\mathrm{GABA}_{\mathrm{A}}$ receptor antagonists. It should be noted that NBQX was also included in the perfusate and this is known to antagonize glycine receptors (Meier and Schmieden, 2003; Lynch, 2004). This may explain the lack of mini or spontaneous IPSCs in the presence of gabazine and NBQX in this, and some previous, studies. Here we measured the glycinergic proportion of ChR2-evoked IPSCs. In instances where we applied strychnine before gabazine ( 4 of 16 neurons), we may overestimate glycine contributions as strychnine is known to also partially block $\mathrm{GABA}_{\mathrm{A}}$ mediated currents at high concentrations (Protti et al., 1997).

The activation of two receptors by SST neurons offers greater level of control at the postsynaptic neuron. Glycine receptors are expressed across the NTS (Dufour et al., 2010) and microinjection of glycine into the NTS decreases arterial pressure and heart rate in anesthetized rats (Talman et al., 1991). Expression of the $\alpha 3$ subunit is prominent (Manzke et al., 2010), and phosphorylation of this subunit, in response to activity of some g-protein coupled receptors (Harvey et al., 2004), can tune glycine receptor function. This implies an additional level of plasticity in the effectiveness of SSTmediated gating. In our recruitment studies, altering the duration of the LED pulses never separated evoked GABAergic from glycinergic IPSCs, suggesting the neurotransmitters were co-released. As activation of presynaptically expressed ChR2 alters neurotransmitter release (Jackman et al., 2014), such a mechanism may have provided the conditions needed to reveal glycinergic activity in the medial NTS.

Thus, SST neurons across the CNS are diverse, here at the level of the NTS they are both inhibitory and excitatory, exhibit ac- 
commodating spiking, and are spontaneously active. SST inhibitory neurons use at least two fast, ionotropic neurotransmitters and are driven by viscerosensory input to affect information processing at the earliest stage of reflex initiation. Autonomic reflexes ensure optimum organ function but they are powerfully modulated to enable coordination with behavioral requirements. Our data, showing an extensive network of SST inputs within the NTS that can gate viscerosensory input, suggests SST inhibitory neurons have the capacity to play a critical role in integrating and modulating viscerosensory information.

\section{References}

Andresen MC, Paton JF (2011) The nucleus of the solitary tract: processing information from viscerosensory afferents. In: Central regulation of autonomic functions (Llewellyn-Smith IJ, Verberne AJ, eds), pp 23-46. London: Oxford UP.

Babic T, Browning KN, Travagli RA (2011) Differential organization of excitatory and inhibitory synapses within the rat dorsal vagal complex. Am J Physiol Gastrointest Liver Physiol 300:G21-32.

Babic T, Ambler J, Browning KN, Travagli RA (2015) Characterization of synapses in the rat subnucleus centralis of the nucleus tractus solitarius. J Neurophysiol 113:466-474.

Bailey TW, Jin YH, Doyle MW, Smith SM, Andresen MC (2006) Vasopressin inhibits glutamate release via two distinct modes in the brainstem. J Neurosci 26:6131-6142.

Bailey TW, Appleyard SM, Jin YH, Andresen MC (2008) Organization and properties of GABAergic neurons in solitary tract nucleus (NTS). J Neurophysiol 99:1712-1722.

Catelli JM, Giakas WJ, Sved AF (1987) GABAergic mechanisms in nucleus tractus solitarius alter blood pressure and vasopressin release. Brain Res 403:279-289.

Chen Z, Travers SP, Travers JB (2016) Inhibitory modulation of optogenetically identified neuron subtypes in the rostral solitary nucleus. J Neurophysiol 116:391-403.

Cinelli E, Iovino L, Bongianni F, Pantaleo T, Mutolo D (2016) GABA(A)and glycine-mediated inhibitory modulation of the cough reflex in the caudal nucleus tractus solitarii of the rabbit. Am J Physiol Lung Cell Mol Physiol 311:L570-580.

Cui Y, Kam K, Sherman D, Janczewski WA, Zheng Y, Feldman JL (2016) Defining preBotzinger complex rhythm- and pattern-generating neural microcircuits in vivo. Neuron 91:602-614.

Doyle MW, Andresen MC (2001) Reliability of monosynaptic sensory transmission in brain stem neurons in vitro. J Neurophysiol 85:22132223.

Duan B, Cheng L, Bourane S, Britz O, Padilla C, Garcia-Campmany L, Krashes M, Knowlton W, Velasquez T, Ren X, Ross S, Lowell BB, Wang Y, Goulding M, Ma Q (2014) Identification of spinal circuits transmitting and gating mechanical pain. Cell 159:1417-1432.

Dufour A, Tell F, Kessler JP, Baude A (2010) Mixed GABA-glycine synapses delineate a specific topography in the nucleus tractus solitarii of adult rat. J Physiol 588:1097-1115.

Fawley JA, Peters JH, Andresen MC (2011) GABA(B)-mediated inhibition of multiple modes of glutamate release in the nucleus of the solitary tract. J Neurophysiol 106:1833-1840.

Fong AY, Stornetta RL, Foley CM, Potts JT (2005) Immunohistochemical localization of GAD67-expressing neurons and processes in the rat brainstem: subregional distribution in the nucleus tractus solitarius. J Comp Neurol 493:274-290.

Han W, Tellez LA, Perkins MH, Perez IO, Qu T, Ferreira J, Ferreira TL, Quinn D, Liu Z-W, Gao XB, Kaelberer MM, Bohórquez DV, Shammah-Lagnado SJ, de Lartigue G, de Araujo IE (2018) A neural circuit for gut-induced reward. Cell 175:665-678.e23.

Harvey RJ, Depner UB, Wässle H, Ahmadi S, Heindl C, Reinold H, Smart TG, Harvey K, Schütz B, Abo-Salem OM, Zimmer A, Poisbeau P, Welzl H, Wolfer DP, Betz H, Zeilhofer HU, Müller U (2004) GlyR alpha3: an essential target for spinal PGE2-mediated inflammatory pain sensitization. Science 304:884-887.

Herman MA, Cruz MT, Sahibzada N, Verbalis J, Gillis RA (2009) GABA signaling in the nucleus tractus solitarius sets the level of activity in dorsal motor nucleus of the vagus cholinergic neurons in the vagovagal circuit. Am J Physiol Gastrointest Liver Physiol 296:G101-G111.
Jackman SL, Beneduce BM, Drew IR, Regehr WG (2014) Achieving high-frequency optical control of synaptic transmission. J Neurosci 34: $7704-7714$.

Janig W (2006) The integrative action of the autonomic nervous system; neurobiology of homeostasis. Cambridge, UK: Cambridge UP.

Jin YH, Bailey TW, Andresen MC (2004) Cranial afferent glutamate heterosynaptically modulates GABA release onto second-order neurons via distinctly segregated metabotropic glutamate receptors. J Neurosci 24:9332-9340.

Kubo T, Kihara M (1987) Evidence for the presence of GABAergic and glycine-like systems responsible for cardiovascular control in the nucleus tractus solitarii of the rat. Neurosci Lett 74:331-336.

Lewin AE, Vicini S, Richardson J, Dretchen KL, Gillis RA, Sahibzada N (2016) Optogenetic and pharmacological evidence that somatostatinGABA neurons are important regulators of parasympathetic outflow to the stomach. J Physiol 594:2661-2679.

Lynch JW (2004) Molecular structure and function of the glycine receptor chloride channel. Physiol Rev 84:1051-1095.

Ma Y, Hu H, Berrebi AS, Mathers PH, Agmon A (2006) Distinct subtypes of somatostatin-containing neocortical interneurons revealed in transgenic mice. J Neurosci 26:5069-5082.

Manzke T, Niebert M, Koch UR, Caley A, Vogelgesang S, Hülsmann S, Ponimaskin E, Müller U, Smart TG, Harvey RJ, Richter DW (2010) Serotonin receptor 1A-modulated phosphorylation of glycine receptor alpha3 controls breathing in mice. J Clin Invest 120:4118-4128.

McDougall SJ, Andresen MC (2012) Low-fidelity GABA transmission within a dense excitatory network of the solitary tract nucleus. J Physiol 590:5677-5689.

McDougall SJ, Guo H, Andresen MC (2017) Dedicated C-fibre viscerosensory pathways to central nucleus of the amygdala. J Physiol 595:901917.

McGarry LM, Packer AM, Fino E, Nikolenko V, Sippy T, Yuste R (2010) Quantitative classification of somatostatin-positive neocortical interneurons identifies three interneuron subtypes. Front Neural Circuits 4:12.

McMenamin CA, Anselmi L, Travagli RA, Browning KN (2016) Developmental regulation of inhibitory synaptic currents in the dorsal motor nucleus of the vagus in the rat. J Neurophysiol 116:1705-1714.

Meier J, Schmieden V (2003) Inhibition of alpha-subunit glycine receptors by quinoxalines. Neuroreport 14:1507-1510.

Michelini LC (2007) The NTS and integration of cardiovascular control during exercise in normotensive and hypertensive individuals. Curr Hypertens Rep 9:214-221.

Okada T, Tashiro Y, Kato F, Yanagawa Y, Obata K, Kawai Y (2008) Quantitative and immunohistochemical analysis of neuronal types in the mouse caudal nucleus tractus solitarius: focus on GABAergic neurons. J Chem Neuroanat 35:275-284.

Ostrowski TD, Ostrowski D, Hasser EM, Kline DD (2014) Depressed GABA and glutamate synaptic signaling by 5 -HT1A receptors in the nucleus tractus solitarii and their role in cardiorespiratory function. J Neurophysiol 111:2493-2504.

Paton JF, Foster WR, Schwaber JS (1993) Characteristic firing behavior of cell types in the cardiorespiratory region of the nucleus tractus solitarii of the rat. Brain Res 604:112-125.

Paton JF, Deuchars J, Li YW, Kasparov S (2001) Properties of solitary tract neurones responding to peripheral arterial chemoreceptors. Neuroscience 105:231-248.

Peters JH, McDougall SJ, Kellett DO, Jordan D, Llewellyn-Smith IJ, Andresen MC (2008) Oxytocin enhances cranial visceral afferent synaptic transmission to the solitary tract nucleus. J Neurosci 28: 11731-11740.

Peters JH, McDougall SJ, Fawley JA, Smith SM, Andresen MC (2010) Primary afferent activation of thermosensitive TRPV1 triggers asynchronous glutamate release at central neurons. Neuron 65:657-669.

Peters JH, McDougall SJ, Fawley JA, Andresen MC (2011) TRPV1 marks synaptic segregation of multiple convergent afferents at the rat medial solitary tract nucleus. PLoS One 6:e25015.

Petilla Interneuron Nomenclature Group (2008) Petilla terminology: nomenclature of features of GABAergic interneurons of the cerebral cortex. Nat Rev Neurosci 9:557-568.

Pfeffer CK, Xue M, He M, Huang ZJ, Scanziani M (2013) Inhibition of 
inhibition in visual cortex: the logic of connections between molecularly distinct interneurons. Nat Neurosci 16:1068-1076.

Potts JT (2002) Neural circuits controlling cardiorespiratory responses: baroreceptor and somatic afferents in the nucleus tractus solitarius. Clin Exp Pharmacol Physiol 29:103-111.

Potts JT (2006) Inhibitory neurotransmission in the nucleus tractus solitarii: implications for baroreflex resetting during exercise. Exp Physiol 91:59-72.

Protti DA, Gerschenfeld HM, Llano I (1997) GABAergic and glycinergic IPSCs in ganglion cells of rat retinal slices. J Neurosci 17:6075-6085.

Saha S, Henderson Z, Batten TF (2002) Somatostatin immunoreactivity in axon terminals in rat nucleus tractus solitarii arising from central nucleus of amygdala: coexistence with GABA and postsynaptic expression of sst2A receptor. J Chem Neuroanat 24:1-13.

Talman WT, Colling JM, Robertson SC (1991) Glycine microinjected into nucleus tractus solitarii of rat acts through cholinergic mechanisms. Am J Physiol 260:H1326-H1331.
Tan W, Janczewski WA, Yang P, Shao XM, Callaway EM, Feldman JL (2008) Silencing preBotzinger complex somatostatin-expressing neurons induces persistent apnea in awake rat. Nat Neurosci 11:538540

Tupal S, Rieger MA, Ling GY, Park TJ, Dougherty JD, Goodchild AK, Gray PA (2014) Testing the role of preBotzinger complex somatostatin neurons in respiratory and vocal behaviors. Eur J Neurosci 40:3067-3077.

Urban-Ciecko J, Barth AL (2016) Somatostatin-expressing neurons in cortical networks. Nat Rev Neurosci 17:401-409.

Vincent A, Tell F (1999) Postnatal development of rat nucleus tractus solitarius neurons: morphological and electrophysiological evidence. Neuroscience 93:293-305.

Wang M, Bradley RM (2010) Properties of GABAergic neurons in the rostral solitary tract nucleus in mice. J Neurophysiol 103:32053218.

Yavorska I, Wehr M (2016) Somatostatin-expressing inhibitory interneurons in cortical circuits. Front Neural Circuits 10:76 